\title{
Processos em meios aleatórios espaço-temporais
}

\author{
Maicon Aparecido Pinheiro
}

TESE APRESENTADA

$\mathrm{AO}$

Instituto de Matemática e Estatística

DA

Universidade DE SÃo PAUlo

PARA

OBTENÇÃO DO TÍTULO

$\mathrm{DE}$

DOUTOR EM CIÊNCIAS

Programa: Estatística

Orientador: Prof. Dr. Luiz Renato Gonçalves Fontes

O presente trabalho foi realizado com apoio da Coordenação de Aperfeiçoamento de Pessoal de Nível Superior - Brasil (CAPES) - Código de Financiamento 001 e do CNPq 


\section{Processos em meios aleatórios espaço-temporais}

Esta versão da tese contém as correções e alterações sugeridas pela Comissão Julgadora durante a defesa da versão original do trabalho, realizada em 31/08/2021. Uma cópia da versão original está disponível no

Instituto de Matemática e Estatística da Universidade de São Paulo.

Comissão Julgadora:

- Prof. Dr. Luiz Renato Gonçalves Fontes (orientador) - IME/USP

- Prof. Dr. Anatoli Iambartsev - IME/USP

- Prof. Dr. Marcelo Richard Hilário - ICEx/UFMG

- Prof. Dra. Maria Eulalia Vares - IM/UFRJ

- Pós-doutor Pablo Almeida Gomes - IME/USP 


\section{Agradecimentos}

De lá pra cá já são vários anos, várias fases... De fase em fase, como num efeito dominó, veio essa tese. Em cada fase sempre houve algo ou alguém por quem sou muito grato. É tanta gente e tanta coisa que não teria condições de espremer os devidos 'obrigados' de modo justo numa seção. É também verdade que dum jeito ou d'outro, uma hora se perde contato; mas a lembrança persiste. Desse modo, uso da oportunidade para agradecer, de modo geral,

Aos que comigo habitaram o bairro Caçador do Meio;

Aos amigos e Professores infantojuvenis, em especial aos da Escola "Paulina de Morais";

Aos funcionários da SABESP de Itapeva no período 2006-2008;

Aos colegas, amigos e professores durante a vivência na FCT-UNESP|USC;

E aos amigos que fiz aqui em SP.

Também manifesto minha gratidão

A todos que tornaram auxílios à permanência estudantil algo possível;

E à CAPES e ao CNPq pelo fomento dado durante o desenvolvimento desse trabalho.

De modo especial, manifesto grande gratidão

À minha família. Sou grato à minha mãe Sant'Ana, meu pai Pedro, meu Irmão Rodrigo e minha irmã Franciane. Não fosse por eles, não teria chego a esse desfecho. Mesmo sob várias adversidades, sempre me deram suporte e carinho. Também agradeço ao meu vô †Zé e minhas avós Isolina e †Tereza.

Ao Professor Renato. Sob sua orientação, consegui superar vários limites pessoais e avançar muito no como fazer matemática. Alguém com um intelecto fora dos padrões e, ainda assim, sempre à disposição e com uma enorme paciência para ensinar.

Por fim, a Deus. 


\section{Resumo}

PINHEIRO, M. A. Processos em meios aleatórios espaço-temporais. 2021. 50 f. Tese (Doutorado) - Instituto de Matemática e Estatística, Universidade de São Paulo, São Paulo, 2021.

Consideramos um passeio aleatório de média nula em $\mathbb{Z}^{d}$ a tempo contínuo num ambiente espaço-temporal em $\mathbb{Z}^{d} \times \mathbb{R}_{+}$dado por processos de nascimento e morte independentes e identicamente distribuídos; de modo que as taxas de salto do passeio, meio-dependentes e dinâmicas no espaço-tempo, são dadas por uma função exponencial decrescente do meio na posição do passeio e assumem valores no conjunto não compacto $(0,1]$. Discorremos sobre o comportamento assintótico do processo, quando o tempo diverge.

Palavras-chave: Passeios Aleatórios, Ambientes Aleatórios Dinâmicos, Processos Estocásticos. 


\section{Abstract}

PINHEIRO, M. A. Processes in space-time random environments. 2021. 50 s. Phd Thesis Instituto de Matemática e Estatística, Universidade de São Paulo, São Paulo, 2021.

We consider a zero mean random walk on $\mathbb{Z}^{d}$ in a space-time environment on $\mathbb{Z}^{d} \times \mathbb{R}_{+}$given by independent and identically distributed birth and death processes; in such a way that the random walk space-time environment-dependent jump rates are given by an exponentially decreasing function of the environment on the current position and assume values in the non-compact set $(0,1]$. We discuss the process asymptotic behavior, when time goes to $+\infty$.

Keywords: Random Walks, Dynamical Random Environments, Stochastic Processes. 


\section{Sumário}

1 Introdução $\quad 1$

1.1 Breve descrição do modelo . . . . . . . . . . . . . . . . . . . . . . . . 1

1.2 Breve descrição dos resultados . . . . . . . . . . . . . . . . . . . 2

1.3 Organização do texto . . . . . . . . . . . . . . . . . . . . . . . 2

2 O modelo $\quad 3$

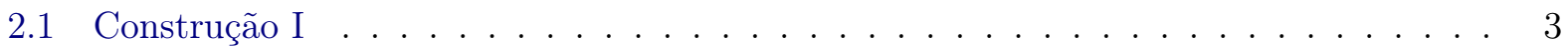

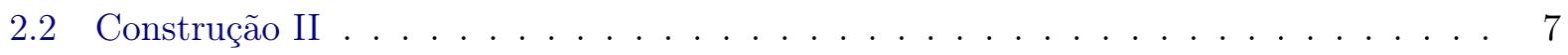

3 Teoremas limites $\quad 9$

3.1 Lei forte dos grandes números . . . . . . . . . . . . . . . . . . . 9

3.2 Teorema central do limite . . . . . . . . . . . . . . . . . . . . . 22

4 Extensões $\quad 25$

4.1 Medida inicial . . . . . . . . . . . . . . . . . . . . . . 25

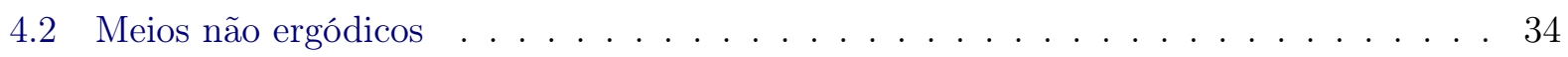

$\begin{array}{lll}5 & \text { Conclusões } & 37\end{array}$

$\begin{array}{ll}\text { Referências Bibliográficas } & 39\end{array}$ 


\section{Capítulo 1}

\section{Introdução}

No processo de aprendizado a respeito de processos aleatórios, somos prontamente introduzidos aos tradicionais passeios aleatórios em $\mathbb{Z}^{d}$. Várias questões típicas acerca desses processos (sobre recorrência, teoremas limites, grandes desvios, entre outras) possuem respostas bem conhecidas e amplamente divulgadas na literatura (vide [Spitzer, 1976], por exemplo).

Os passeios aleatórios em $\mathbb{Z}^{d}$ fornecem modelos simples e convencionais para a difusão de uma partícula sobre um determinado meio. Nesses modelos, há a suposição de que o mecanismo de transição da partícula seja regido por uma distribuição de probabilidade meio-independente. No entanto, na prática, é muito comum que o meio sobre o qual a partícula evolui seja altamente irregular, por conta de defeitos, impurezas, flutuações, etc; e é razoável supor que tais irregularidades exerçam alguma influência na partícula. Nesse sentido, uma extensão aos passeios aleatórios em $\mathbb{Z}^{d}$ são os passeios aleatórios em ambientes aleatórios em $\mathbb{Z}^{d}$.

De modo informal, passeios aleatórios em ambientes aleatórios em $\mathbb{Z}^{d}$ são passeios aleatórios em $\mathbb{Z}^{d}$ cujo núcleo de transição depende de um processo aleatório $\omega$ denominado ambiente aleatório. Esse ambiente aleatório $\omega$, por sua vez, pode ser classificado em estático - é escolhido ao acaso no tempo zero e permanece fixo ao longo do tempo - ou dinâmico - se altera no tempo segundo alguma dinâmica estocástica (para maiores detalhes, recomendamos [Avena, 2010], Capítulo 1). Em contraste aos passeios aleatórios usuais, os passeios aleatórios em ambientes aleatórios - a depender de $\omega$ - podem não exibir um Teorema Central do Limite, por exemplo. Nesse trabalho, apresentamos uma contribuição sob o caso em que $\omega$ é dinâmico; tópico que têm sido intensivamente estudado sob diversas condições nos últimos trinta anos (Boldrighini et al. [1992], Bandyopadhyay e Zeitouni [2006], Boldrighini et al. [2007], Dolgopyat et al. [2008], Dolgopyat e Liverani [2009], Yilmaz [2009], Redig e Völlering [2013], entre outros). Apresentamos a seguir uma breve descrição do modelo e dos nossos resultados, bem como a organização do texto.

\subsection{Breve descrição do modelo}

O modelo é um processo de Markov $(X(t), \omega(t))_{t \in \mathbb{R}_{+}}$em que $\omega(\cdot)=\left(\omega_{\mathbf{x}}(\cdot)\right)_{\mathbf{x} \in \mathbb{Z}^{d}} \operatorname{com}\left(\omega_{\mathbf{x}}(t)\right)_{t \in \mathbb{R}_{+}}$, $\mathbf{x} \in \mathbb{Z}^{d}$, processos de nascimento e morte em $S=\mathbb{N}$ independentes com taxa de nascimento $p \leqslant 1 / 2$ e taxa de morte $q=1-p>p$; e $(X(t))_{t \in \mathbb{R}_{+}}$um passeio aleatório de média nula a tempo contínuo em $\mathbb{Z}^{d}$ cuja taxa de salto em $\mathbf{x} \in \mathbb{Z}^{d}$ no tempo $t \in \mathbb{R}_{+}$é dada por $\lambda_{\mathbf{x}}(t)=a^{-\omega_{\mathbf{x}}(t)}, a>1$. De acordo com a literatura, vamos chamar $(X(t))_{t \in \mathbb{R}_{+}}$de passeio aleatório em $\mathbb{Z}^{d}$ a tempo contínuo no ambiente/meio aleatório dinâmico $(\omega(t))_{t \in \mathbb{R}_{+}}$. Também iremos nos referir a $(\omega(t))_{t \in \mathbb{R}_{+}}$, de modo 
abreviado, por ambiente/meio e a $X(t)$ por posição da partícula no tempo $t$. Para uma descrição detalhada do modelo veja o segundo capítulo do trabalho, onde apresentamos duas construções do processo.

\subsection{Breve descrição dos resultados}

Inicialmente, mostramos - via uma aplicação do Teorema Ergódico Subaditivo (vide [Durret, 2010], Capitulo 7, Seção 4, Teorema 7.4.1) - uma Lei dos Grandes Números para os tempos de salto de $(X(t))_{t \in \mathbb{R}_{+}}$a partir de $\omega(0) \equiv \mathbf{0}$. Para a verificação das condições do Teorema Ergódico Subaditivo, provamos - a partir das particularidades do modelo - uma dominação estocástica invariante no tempo para a lei de $\omega$. Daí - via argumentos usuais da teoria de processos de renovação - garantimos a validade de um Teorema Central do Limite para $(X(t))_{t \in \mathbb{R}_{+}}$sob $\omega(0) \equiv \mathbf{0}$. Na sequência, com o uso de técnicas de acoplamento, estendemos os resultados anteriores para uma classe mais ampla de medidas iniciais para o ambiente. Findamos discorrendo sobre o comportamento assintótico de $(X(t))_{t \in \mathbb{R}_{+}}$no cenário em que $\left(\omega_{\mathbf{x}}(t)\right)_{t \in \mathbb{R}_{+}}, \mathbf{x} \in \mathbb{Z}^{d}$, são não ergódicos.

No caso em que $S$ é finito (ou compacto), versões dos resultados acima podem ser encontradas na literatura (veja, por exemplo, [Dolgopyat et al., 2008], [Dolgopyat e Liverani, 2009] e [Redig e Völlering, 2013]). O caso sob consideração não foi estudado, pois $(\omega(t))_{t \in \mathbb{R}_{+}}$tem como espaço de estados o conjunto não compacto $\mathbb{N}^{\mathbb{Z}^{d}}$ e a questão sobre existência e unicidade de uma medida estacionária para o ambiente visto pela partícula (técnica utilizada sob a compacidade) permanece aberta.

\subsection{Organização do texto}

Como já mencionado, segue no próximo capítulo a construção do modelo. No capítulo 3, expomos os teoremas limites para a posição da partícula sob $\omega(0) \equiv \mathbf{0}$. No quarto capítulo, exibimos uma extensão dos resultados para uma classe de medidas iniciais para $\omega$ e discorremos sobre o caso em que o meio é definido a partir de processos não ergódicos. Por fim, no capítulo 5, apresentamos nossas conclusões. 


\section{Capítulo 2}

\section{O modelo}

Nesse trabalho, denotamos por $\mathcal{D}\left(\mathbb{R}_{+}, S\right), S \subset \mathbb{R}^{d}, d \in \mathbb{N}_{*}, \mathbb{N}_{*}:=\mathbb{N} \backslash\{0\}$, o conjunto das trajetórias càdlàg de $\mathbb{R}_{+}$em $S$; com $a:=b(a=: b)$ usado para dizer que $a$ é definido por $b$ ( $b$ é definido por $a$ ). Por $\mathbf{0} \in E$ e $\mathbf{1} \in E, E=\mathbb{N}^{d}, \mathbb{Z}^{d}, \mathbb{N}^{\mathbb{Z}^{d}}$, representamos, respectivamente, o elemento nulo e o elemento com todas as coordenadas iguais a 1. Também usaremos $M \sim P N M(\lambda, \mu), \lambda, \mu \in \mathbb{R}_{+}$, para indicar que $M$ é um processos de nascimento e morte com taxa de nascimento $\lambda$ e taxa de morte $\mu$. Dito isso, vamos apresentar duas construção ao modelo. A partir da primeira construção, que nos parece a mais natural, derivaremos a segunda. Esta, por sua vez, trará simplificações que serão essenciais para a prova - no próximo capítulo - de nossos principais resultados.

\subsection{Construção I}

Seja $\omega(\cdot)=\left(\omega_{\mathbf{x}}(\cdot)\right)_{\mathbf{x} \in \mathbb{Z}^{d}}$ uma família de processos de nascimento e morte em $S=\mathbb{N}$ independentes com taxa de nascimento $p \in(0,1 / 2)$ e taxa de morte $q=1-p>p$, cada um a partir da respectiva distribuição inicial $\mu_{\mathbf{x}, 0}$ (representaremos por $\mu_{\mathbf{x}, t}$ a distribuição de $\omega_{\mathbf{x}}(t), t \in \mathbb{R}_{+}$), $\mathbf{x} \in \mathbb{Z}^{d}$. Denominamos $\omega:=\omega(\cdot)$ por ambiente/meio aleatório dinâmico ou, de modo abreviado, ambiente/meio. A perspectiva adotada de $\omega$, que pode ser visto como um campo aleatório $\left\{\omega_{\mathbf{x}}(t):(\mathbf{x}, t) \in \mathbb{Z}^{d} \times \mathbb{R}_{+}\right\}$, é a de um processo estocástico $(\omega(t))_{t \in \mathbb{R}_{+}}$a tempo contínuo no espaço de estados $\Lambda:=S^{\mathbb{Z}^{d}}$ com distribuição inicial $\hat{\mu}_{0}:=\bigotimes_{\mathbf{x} \in \mathbb{Z}^{d}} \mu_{\mathbf{x}, 0}\left(\hat{\mu}_{t}:=\bigotimes_{\mathbf{x} \in \mathbb{Z}^{d}} \mu_{\mathbf{x}, t}, t \in \mathbb{R}_{+}\right)$e com espaço de trajetórias dado por $A:=\mathcal{D}\left(\mathbb{R}_{+}, \mathbb{N}\right)^{\mathbb{Z}^{d}}$. Denotemos a lei de $\omega$ por $\mathrm{P}_{\hat{\mu}_{0}}$. Além disso, independente de $\omega$, seja $\pi$ uma medida de probabilidade em $\mathbb{Z}^{d} \backslash\{\mathbf{0}\}$ tal que $\sum_{\mathbf{x} \in \mathbb{Z}^{d}} \mathbf{x} \pi(\mathbf{x})=\mathbf{0}, \sum_{\mathbf{x} \in \mathbb{Z}^{d}}\|\mathbf{x}\|^{2} \pi(\mathbf{x})<+\infty$, com $\|\cdot\|$ a norma euclideana, e consideremos $\xi:=\left\{\xi_{n}\right\}_{n \in \mathbb{N}_{*}}$ uma sequência de vetores aleatórios independentes e identicamente distribuídos (i.i.d.) em $\mathbb{Z}^{d}$ com distribuição $\pi$.

Agora, seja $\mathcal{M}$ um processo pontual de Poisson de taxa 1 em $\mathbb{R}^{d} \times \mathbb{R}_{+}$independente de $\omega$ e $\xi$ e, para cada $\mathbf{x}=\left(x_{1}, \ldots, x_{d}\right) \in \mathbb{Z}^{d}$, definamos

$$
\mathcal{M}_{\mathbf{x}}=\mathcal{M} \cap\left(Q_{\mathbf{x}} \times \mathbb{R}_{+}\right),
$$


em que $Q_{\mathbf{x}}=\underset{i=1}{\stackrel{d}{\times}}\left[q_{x_{i}}, q_{x_{i}}+1\right)$, com $q_{x_{i}}:=x_{i}-1 / 2,1 \leqslant i \leqslant d$. É claro que

$$
\mathcal{M}=\bigcup_{\mathbf{x} \in \mathbb{Z}^{d}} \mathcal{M}_{\mathbf{x}}
$$

e que, pelas propriedades bem conhecidas de processos de Poisson, $\left\{\mathcal{M}_{\mathbf{x}}: \mathbf{x} \in \mathbb{Z}^{d}\right\}$ é uma coleção de processos independentes, com $\mathcal{M}_{\mathbf{x}}$ um processo pontual de Poisson com taxa 1 em $Q_{\mathbf{x}} \times[0,+\infty)$. Dados $\omega \in A$ e $a>1$, façamos

$$
\mathcal{N}_{\mathbf{x}}=\left\{\left(y_{1}, \ldots, y_{d}, r\right) \in \mathcal{M}_{\mathbf{x}}: y_{d} \in\left[q_{x_{d}}, q_{x_{d}}+a^{-\omega_{\mathbf{x}}(r)}\right)\right\}, \quad \mathbf{x} \in \mathbb{Z}^{d}
$$

Note que a projeção de $\mathcal{N}_{\mathbf{x}}$ em $\{\mathbf{x}\} \times \mathbb{R}_{+}$é um processo pontual de Poisson não homogêneo em $\{\mathbf{x}\} \times \mathbb{R}_{+}$com função de intensidade

$$
\lambda_{\mathbf{x}}(r)=a^{-\omega_{\mathbf{x}}(r)}, \quad \mathbf{x} \in \mathbb{Z}^{d}, r \geqslant 0 .
$$

Fixemos $X(0)=\mathbf{x}_{0}, \mathbf{x}_{0} \in \mathbb{Z}^{d}$. Definamos $X(t), t \in \mathbb{R}_{+}$, da seguinte maneira:

Seja $\tau_{0}=0$ e definamos

$$
\tau_{1}=\inf \left\{r>0: \mathcal{N}_{\mathbf{x}_{0}} \cap\left(Q_{\mathbf{x}_{0}} \times(0, r]\right) \neq \varnothing\right\}
$$

com a convenção de que inf $\varnothing=+\infty$. Para todo $t \in\left(0, \tau_{1}\right), X(t)=X(0)$ e

$$
X\left(\tau_{1}\right)=X(0)+\xi_{1} .
$$

Para $n \geqslant 2$, suponhamos definidos $\tau_{1}, \ldots, \tau_{n-1}$ e $X(t)$, para $t \leqslant \tau_{n-1}$, e definamos

$$
\tau_{n}=\inf \left\{r>\tau_{n-1}: \mathcal{N}_{X_{\tau_{n-1}}} \cap\left(Q_{X_{\tau_{n-1}}} \times\left(\tau_{n-1}, r\right]\right) \neq \varnothing\right\}
$$

Para $t \in\left(\tau_{n-1}, \tau_{n}\right), X(t)=X\left(\tau_{n-1}\right)$ e

$$
X\left(\tau_{n}\right)=X\left(\tau_{n-1}\right)+\xi_{n}
$$

Em palavras, $\left(\tau_{n}\right)_{n \in \mathbb{N}}$ são os tempos de salto do processo $(X(t))_{t \in \mathbb{R}_{+}}$, que por sua vez, dado $\omega \in A$, é um passeio aleatório a tempo contínuo em $\mathbb{Z}^{d}$ a partir de $\mathbf{x}_{0}$ com taxa de salto em $\mathbf{x}$ no tempo $t$ dada por $a^{-\omega_{\mathbf{x}}(t)}, a>1, \mathbf{x} \in \mathbb{Z}^{d}$. Além disso, se a posição do passeio é $\mathbf{x}$, o próximo sítio a ser visitado é dado por $\mathbf{x}+\mathbf{y}$, com $\mathbf{y}$ gerado a partir de $\pi, \mathbf{x}, \mathbf{y} \in \mathbb{Z}^{d}$. O conjunto de possíveis realizações para $(X(t))_{t \in \mathbb{R}_{+}}$é dado por $\mathcal{D}\left(\mathbb{R}_{+}, \mathbb{Z}^{d}\right)$. Denotemos por $P_{\mathbf{x}_{0}}^{\omega}$ a lei de $(X(t))_{t \in \mathbb{R}_{+}}$dado $\omega \in A$. Observamos que, pelo fato de $\mathcal{N}_{\mathbf{x}} \subset \mathcal{M}_{\mathbf{x}}$ para todo $\mathbf{x} \in \mathbb{Z}^{d}$, segue pelas propriedades bem conhecidas de falta de memória de processos de Poisson que, para cada $n \in \mathbb{N}_{*}, P_{\mathbf{x}_{0}}^{\omega}$ quase certamente $\left(P_{\mathbf{x}_{0}}^{\omega}-\right.$ q.c. $), \tau_{n}-\tau_{n-1} \geqslant Z_{n}$, em que $Z_{n}$ é uma exponencial padrão. Assim, $\tau_{n} \nearrow+\infty$ $P_{\mathbf{x}_{0}}^{\omega}$-q.c. quando $n \nearrow+\infty$, isto é (i.e.), o processo não é explosivo. Logo, dado $\omega \in A$, podemos construir o processo $(X(t))_{t \in \mathbb{R}_{+}}$por indução e $X(t)$ está bem definido para todo $t \in \mathbb{R}_{+}$. 
O modelo é então o processo de Markov $(X(t), \omega(t))_{t \in \mathbb{R}_{+}}$, com $\omega(t)=\left(\omega_{\mathbf{x}}(t)\right)_{\mathbf{x} \in \mathbb{Z}^{d}}$ o ambiente aleatório dinâmico já descrito acima e $(X(t))_{t \in \mathbb{R}_{+}}$um processo estocástico em $\mathbb{Z}^{d}$ a tempo contínuo a partir de $\mathbf{x}_{0}$ cuja cadeia imersa, digamos $\left(\mathrm{x}_{n}\right)_{n \in \mathbb{N}}$, é um passeio aleatório de média nula a tempo discreto em $\mathbb{Z}^{d}$ com incrementos distribuídos de acordo com $\pi$. O ambiente em cada $\mathbf{x} \in \mathbb{Z}^{d}$ evolui como uma cópia independente de um processo de Markov ergódico em $\mathbb{N}$, com distribuição invariante

$$
\nu(k)=(1-\rho) \rho^{k}, \quad k \in \mathbb{N}, \quad \rho=p / q<1 .
$$

As taxas de salto para $(X(t))_{t \in \mathbb{R}_{+}}$em $\mathbf{x} \in \mathbb{Z}^{d}$ no tempo $t$ são dadas por

$$
\lambda_{\mathbf{x}, \omega}(t)=a^{-\omega_{\mathbf{x}}(t)}, \quad a>1
$$

Escolhemos (2.10) a fim de destacar o efeito da dinamicidade de $\omega$ no tempo sobre o comportamento assintótico da partícula. De fato, suponhamos $\hat{\mu}_{0}:=\bigotimes_{x \in \mathbb{Z}^{d}} \nu$, por exemplo. A versão análoga para o modelo com o meio estático consiste de $(\mathcal{X}, \tau)$; um Passeio Aleatório com Taxas Aleatórias em $\mathbb{Z}^{d}$ (veja [Fontes et al., 2002], Definição 1.1.) em que $\mathcal{X}$ e $X$ possuem a mesma cadeia imersa e $\tau=\left(\tau_{\mathbf{x}}\right)_{\mathbf{x} \in \mathbb{Z}} \operatorname{com}$

$$
\tau_{\mathbf{x}}=a^{\omega_{\mathbf{x}}(0)}, \quad \mathbf{x} \in \mathbb{Z}^{d} .
$$

Pode-se mostrar que para $a<q / p\left(\tau_{x}\right.$ têm primeiro momento finito), vale (para quase todo $\tau$ ) um Teorema Central do Limite para $\mathcal{X}$ enquanto que, para $a \geqslant q / p, \mathcal{X}$ é sub-difusivo. Por outro lado, como era razoável esperar (iremos provar posteriormente), vale o Teorema Central do Limite para $X$ qualquer que seja $a>1$. Em palavras, o fato de $\omega$ ter como componentes processos recorrentes positivos impede a formação de regiões no espaço $\mathbb{Z}^{d}$ nas quais a partícula apresente desaceleração suficiente no deslocamento para inviabilizar a validade do Teorema Central do Limite, independente do valor do parâmetro $a$.

Para $f: \mathbb{Z}^{d} \times \Lambda \rightarrow \mathbb{R}$ contínua, limitada e cilíndrica (depende apenas de um número finito de coordenadas de $\left.(\mathbf{x}, \omega) \in \mathbb{Z}^{d} \times \Lambda\right)$, o gerador do processo em $s \in \mathbb{R}_{+}$é

$$
\mathcal{L}^{s} f(\mathbf{x}, \omega)=\lambda_{\mathbf{x}, \omega}(s) \sum_{\mathbf{z} \in \mathbb{Z}^{d}} \pi(\mathbf{z}-\mathbf{x})[f(\mathbf{z}, \omega)-f(\mathbf{x}, \omega)]+\mathcal{L}^{*} f(\mathbf{x}, \omega)
$$

em que $\mathcal{L}^{*}$ é o gerador do processo de ambiente livre, nesse caso dado por

$$
\mathcal{L}^{*} f(\mathbf{x}, \omega)=\sum_{\mathbf{x} \in \mathbb{Z}^{d}} \sum_{e \in\{-1,1\}} c\left(\omega_{\mathbf{x}}, e\right)\left[f\left(\mathbf{x}, \omega^{\mathbf{x}, e}\right)-f(\mathbf{x}, \omega)\right],
$$

onde $c(a, b): \mathbb{N} \times\{-1,+1\} \rightarrow[0,1] \operatorname{com} c(0,1)=p, c(0,-1)=0, c(y, 1)=1-c(y,-1)=p$, $y>0$, e $\omega_{\mathbf{z}}^{\mathbf{x}, e}=\omega_{\mathbf{z}}+e \mathbb{1}\{\mathbf{z}=\mathbf{x}\}, \mathbf{z} \in \mathbb{Z}^{d}$. O respectivo espaço amostral fica então dado por $\Omega=\mathcal{D}\left(\mathbb{R}_{+}, \mathbb{Z}_{+}\right)^{\mathbb{Z}^{d}} \times \mathcal{D}\left(\mathbb{R}_{+}, \mathbb{Z}^{d}\right)$. Denotemos por $\left(\Omega, \mathcal{F}, \mathbf{P}_{\hat{\mu}_{0}, \mathbf{x}_{0}}\right)$ o espaço de probabilidade para o modelo. A fim de fixar a construção, ressaltamos que a primeira marginal de $\mathbf{P}_{\hat{\mu}_{0}, \mathbf{x}_{0}}$ é dada pela lei do ambiente $\mathrm{P}_{\hat{\mu}_{0}}$, e a lei condicional da segunda marginal dado $\omega \in A$, é dada por $P_{\mathbf{x}_{0}}^{\omega}$. Em suma,

$$
\mathbf{P}_{\hat{\mu}_{0}, \mathbf{x}_{0}}(M \times N)=\int_{M} \mathrm{dP}_{\hat{\mu}_{0}}(\omega) P_{\mathbf{x}_{0}}^{\omega}(N),
$$


em que $M$ e $N$ são subconjuntos mensuráveis de $A$ e $\mathcal{D}\left(\mathbb{R}_{+}, \mathbb{Z}^{d}\right)$, respectivamente. De acordo com a literatura, vamos chamar a lei $P_{\mathbf{x}_{0}}^{\omega}$ de lei quenched e a lei $\mathbf{P}_{\hat{\mu}_{0}, \mathbf{x}_{0}}$ de lei annealed. Diremos que uma afirmação sobre o passeio aleatório $(X(t))_{t \in \mathbb{R}_{+}}$vale $\mathbf{P}_{\mathbf{x}_{0}, \hat{\mu}_{0}}-$ q.c. se $\mathrm{P}_{\hat{\mu}_{0}}$ - para quase todo $\left(\mathrm{P}_{\hat{\mu}_{0}}-\right.$ q.t. $) \omega$, a afirmação vale $P_{\mathbf{x}_{0}}^{\omega}-$ q.c.. De modo respectivo, vamos denotar por $\mathrm{E}_{\hat{\mu}_{0}}(\cdot), E_{\mathbf{x}_{0}}^{\omega}(\cdot)$ e $\mathbf{E}_{\hat{\mu}_{0}, \mathbf{x}_{0}}(\cdot)$ as esperanças com respeito a $\mathrm{P}_{\hat{\mu}_{0}}, P_{\mathbf{x}_{0}}^{\omega}$ e $\mathbf{P}_{\hat{\mu}_{0}, \mathbf{x}_{0}}$. Além disso, no que segue, sem perda de generalidade, $\mathbf{x}_{0} \equiv \mathbf{0}$, e omitiremos tal índice, i.e.,

$$
P^{\omega}:=P_{\mathbf{0}}^{\omega} \quad \text { e } \quad \mathbf{P}_{\hat{\mu}_{0}}:=\mathbf{P}_{\hat{\mu}_{0}, \mathbf{0}} .
$$

Também omitiremos o índice $\hat{\mu}_{0}$ quando este não for relevante. Com isso claro, a partir de agora, indicaremos por

$$
\mathbf{P}_{\mathbf{w}}, \quad \mathbf{w} \in \Lambda,
$$

a lei do processo a partir de $\omega(0)=\mathbf{w}$ e $\mathbf{x}_{0} \equiv \mathbf{0}$. Façamos ainda $\Delta_{n}:=\tau_{n}-\tau_{n-1}, n \in \mathbb{N}_{*}$. Ressaltamos que, por construção (vide Equação 2.13),

$$
\mathbf{P}_{\hat{\mu}_{0}}\left(\tau_{1}>t\right)=\mathrm{E}_{\hat{\mu}_{0}}\left[\exp \left(-\int_{0}^{t} a^{-\omega_{\mathbf{0}}(s)} d s\right)\right], \quad t \in \mathbb{R}_{+},
$$

e, para $n \in \mathbb{N}$, sob $\tau_{n}<+\infty$,

$$
\mathbf{P}_{\hat{\mu}_{0}}\left(\Delta_{n+1}>t\right)=\mathrm{E}_{\hat{\mu}_{0}}\left[\exp \left(-\int_{\tau_{n}}^{\tau_{n}+t} a^{-\omega_{x_{n}}(s)} d s\right)\right], \quad t \in \mathbb{R}_{+},
$$

relembrando que $\left(\mathrm{x}_{n}\right)_{n \in \mathbb{N}}$ indica a cadeia imersa de $(X(t))_{t \in \mathbb{R}_{+}}$. Por conveniência na notação, para $n \in \mathbb{N}$, façamos,

$$
I_{n}(t):=\int_{\tau_{n}}^{\tau_{n}+t} a^{-\omega_{x_{n}}(s)} d s, \quad t \in \mathbb{R}_{+} .
$$

Dado $\tau_{n}<+\infty, I_{n}: \mathbb{R}_{+} \rightarrow \mathbb{R}_{+}, n \in \mathbb{N}$, está bem definida e é inversível $\mathbf{P}-$ q.c.. De fato, por construção, o meio é definido a partir de processos não explosivos. Isto garante $\mathbf{P}-$ q.c. que, para $0 \leqslant s<t<\infty$, a função limitada $\lambda: \mathbb{R}_{+} \rightarrow(0,1]$ dada por $\lambda(x)=a^{-\omega_{x_{n}}(x)}, x \in \mathbb{R}_{+}, a>1$, tem um número finito de descontinuidades em $[s, t]$, i.e., é integrável em $[s, t]$ (vide [Lima, 2010], Capítulo IX, Corolário 2, pg 320). A inversibilidade de $I_{n}(t)$ segue do fato de $I_{n}(t)$ ser estritamente crescente e contínua, o que pode ser verificado sem maiores dificuldades, uma vez que $\lambda$ é limitada e positiva. Assim, (2.16) e (2.17) podem ser reescritas, respectivamente, como

$$
\mathbf{P}_{\hat{\mu}_{0}}\left(\tau_{1}>t\right)=\mathrm{E}_{\hat{\mu}_{0}}\left[e^{-I_{0}(t)}\right], \quad t \in \mathbb{R}_{+},
$$

e, para $n \in \mathbb{N}$, sob $\tau_{n}<+\infty$,

$$
\mathbf{P}_{\hat{\mu}_{0}}\left(\Delta_{n+1}>t\right)=\mathrm{E}_{\hat{\mu}_{0}}\left[e^{-I_{n}(t)}\right], \quad t \in \mathbb{R}_{+}
$$

Vale ressaltar que $\tau_{n}<+\infty, \mathbf{P}_{\hat{\mu}_{0}}-q . c ., n \in \mathbb{N}$. De fato, por indução, $\tau_{0} \equiv 0<+\infty$ e, supondo $\tau_{n}<+\infty, n \in \mathbb{N}$,

$$
\mathbf{P}_{\hat{\mu}_{0}}\left(\Delta_{n+1}=+\infty\right)=\lim _{k \nearrow+\infty} \mathrm{E}_{\hat{\mu}_{0}}\left[e^{-I_{n}(k)}\right]=\mathrm{E}_{\hat{\mu}_{0}}\left[\exp \left(-\int_{\tau_{n}}^{\infty} a^{-\omega_{x_{n}}(s)} d s\right)\right],
$$


com a segunda passagem decorrente de uma aplicação direta do Teorema da Convergência Dominada (vide [Durret, 2010], Teorema 1.6.7); e

$$
\mathrm{E}_{\hat{\mu}_{0}}\left[\exp \left(-\int_{\tau_{n}}^{\infty} a^{-\omega_{x_{n}}(s)} d s\right)\right] \leqslant \mathrm{E}_{\hat{\mu}_{0}}\left[\exp \left(-\int_{\tau_{n}}^{\infty} \mathbb{1}\left\{\omega_{x_{n}}(s)=0\right\} d s\right)\right]=0,
$$

uma vez que a integral no termo central da expressão acima é infinita $\mathrm{P}_{\hat{\mu}_{0}}-$ q.c., pela recorrência positiva de $\omega_{x_{n}}$.

\subsection{Construção II}

Findamos o capítulo com uma construção alternativa do modelo.

Façamos $\mathrm{T}_{0}=0$ e, para $n \in \mathbb{N}_{*}, \mathrm{~T}_{n}=\sum_{k=0}^{n-1} I_{k}\left(\Delta_{k+1}\right)$.

Lema 2.1. $\left\{\mathrm{T}_{n}: n \in \mathbb{N}_{*}\right\}$ é um processo pontual de Poisson de taxa 1 em $\mathbb{R}_{+}$e independe de $\omega$ e $\xi, \mathbf{P}-q . c .$.

Demonstração. Equivale a mostrar $\left(I_{n}\left(\Delta_{n+1}\right)\right)_{n \in \mathbb{N}}$ são exponenciais padrões independentes entre si e independentes de $\omega$ e $\xi, \mathbf{P}_{\hat{\mu}_{0}}-q . c$., $\hat{\mu}_{0}$ genérica. Para a independência, basta notar que, para quaisquer $\omega$ e $\xi$ fixados, $\left(\Delta_{n}\right)_{n \in \mathbb{N}_{*}}$ são os tempos entre ocorrências de marcas em um processo de Poisson não homogêneo bem definido, que são independentes entre si. E, além disso,

$$
\mathbf{P}\left(I_{n}\left(\Delta_{n+1}\right)>t\right)=\mathbf{P}\left(\Delta_{n+1}>I_{n}^{-1}(t)\right)=\mathrm{E}\left[e^{-I_{n}\left(I_{n}^{-1}(t)\right)}\right]=e^{-t}, t \in \mathbb{R}_{+} .
$$

Como $\Delta_{n+1}=I_{n}^{-1}\left(I_{n}\left(\Delta_{n+1}\right)\right)=I_{n}^{-1}\left(\mathrm{~T}_{n+1}-\mathrm{T}_{n}\right), n \in \mathbb{N}$, e, pelo Lema $2.1,\left\{\mathrm{~T}_{n}: n \in \mathbb{N}\right\}$ consiste de um processo pontual de Poisson de taxa 1 em $\mathbb{R}_{+}$independente de $\omega$ e $\xi, \mathbf{P}-$ q.c., decorre a seguinte construção alternativa para o modelo, distinta da primeira pelo modo como são definidos os tempos de saltos.

Sejam $\omega, \xi$ como descritos no início do capítulo. Além disso, seja $\mathrm{V}=\left(\mathrm{V}_{n}\right)_{n \in \mathbb{N}}$ um sequência de exponenciais padrões independentes. Então, dados $\omega$, podemos construir o processo $(X(t))_{t \in \mathbb{R}_{+}}$da seguinte maneira:

Façamos $X(0)=\mathrm{x}_{0} \equiv \mathbf{0}$ e $\tau_{0}=0$. Definamos

$$
\tau_{1}=I_{0}^{-1}\left(\mathrm{~V}_{1}\right)
$$

Para todo $t \in\left(0, \tau_{1}\right), X(t)=X(0)$ e

$$
X\left(\tau_{1}\right)=X(0)+\xi_{1}=\mathrm{x}_{1} .
$$


Para $n \geqslant 2$, suponhamos definidos $\tau_{1}, \ldots, \tau_{n-1}$ e $X(t)$, para $t \leqslant \tau_{n-1}$, e definamos

$$
\tau_{n}=\tau_{n-1}+I_{n-1}^{-1}\left(\mathrm{~V}_{n}\right)
$$

Para $t \in\left(\tau_{n-1}, \tau_{n}\right), X(t)=X\left(\tau_{n-1}\right) \mathrm{e}$

$$
X\left(\tau_{n}\right)=X\left(\tau_{n-1}\right)+\xi_{n}=\mathbf{x}_{n} .
$$

Por indução, o processo fica bem definido.

Antes, na primeira construção, utilizamos as marcas de uma coleção $\mathcal{N}:=\left\{\mathcal{N}_{\mathbf{x}}: \mathbf{x} \in \mathbb{Z}^{d}\right\}$ de processos pontuais $\omega$-dependentes como candidatas aos tempos de salto da partícula; cujos destinos eram regidos independentemente pela sequência $\xi=\left(\xi_{n}\right)_{n \in \mathbb{N}_{*}}$. Na Construção II, toda informação útil à formação dos tempos de saltos - antes oriunda de $\omega$ e $\mathcal{N}$ - é dada por $\omega$ e $V$, que são independentes entre si. Assim, a partir de três elementos independentes - $\omega, \vee$ e $\xi$ - somos capazes de construir o processo. No próximo capítulo, usaremos a Construção II para definir um acoplamento essencial à prova de uma Lei Forte dos Grandes Números para os tempos de salto da partícula. 


\section{Capítulo 3}

\section{Teoremas limites}

Os principais resultados nesse capítulo são, sob $\mathbf{P}_{\mathbf{0}}{ }^{1}$, uma Lei Forte dos Grandes Números e um Teorema Central do Limite para a posição da partícula.

\subsection{Lei forte dos grandes números}

Derivaremos a Lei Forte dos Grandes Números para $(X(t))_{t \in \mathbb{R}_{+}}$a partir de uma Lei Forte dos Grandes Números para $\left(\tau_{n}\right)_{n \in \mathbb{N}}$, ambas sob $\mathbf{P}_{\mathbf{0}}$. Antes da prova dessa última, dada na Proposição 3.1, vamos introduzir alguns resultados preliminares e necessários.

Definição 3.1. Dadas $v_{1}$ e $v_{2}$ medidas de probabilidade em $\mathbb{N}$, denotaremos por $v_{1} \leq v_{2}$ a dominação estocástica de $v_{1}$ por $v_{2}$, i.e.,

$$
v_{1}\left(\mathbb{N} \backslash \mathrm{A}_{k}\right) \leqslant v_{2}\left(\mathbb{N} \backslash \mathrm{A}_{k}\right), \quad \mathrm{A}_{k}:=\{0, \ldots, k\}, \forall k \in \mathbb{N}_{*}
$$

Escreveremos $v_{1}<v_{2}$ quando a desigualdade acima for estrita. No mesmo sentido, dadas $\left(v_{1, \mathbf{x}}\right)_{\mathbf{x} \in \mathbb{Z}^{d}}$, $\left(v_{2, \mathbf{x}}\right)_{\mathbf{x} \in \mathbb{Z}^{d}}$ medidas de probabilidade em $\mathbb{N}, \hat{v}_{1}:=\bigotimes_{\mathbf{x} \in \mathbb{Z}^{d}} v_{1, \mathbf{x}}$ e $\hat{v}_{2}:=\bigotimes_{\mathbf{x} \in \mathbb{Z}^{d}} v_{2, \mathbf{x}}$ medidas de probabilidade em $\mathbb{N}^{d}$, escreveremos $\hat{v}_{1} \leq \hat{v}_{2}$ quando $v_{1, \mathbf{x}} \leq v_{2, \mathbf{x}}$ para todo $\mathbf{x} \in \mathbb{Z}^{d}$. Se $\hat{v}_{1} \leq \hat{v}_{2}$ e $v_{1, \mathbf{x}}<v_{2, \mathbf{x}}$ para algum $\mathbf{x} \in \mathbb{Z}^{d}$, utilizaremos a notação $\hat{v}_{1}<\hat{v}_{2}$.

Indicaremos por $\hat{\nu}$ a medida produto para o meio $\omega$ dada por

$$
\hat{\nu}=\underset{\mathbf{x} \in \mathbb{Z}^{d}}{\bigotimes} \nu
$$

recordando que

$$
\nu(k)=(1-\rho) \rho^{k}, \quad k \in \mathbb{N}, \quad \rho=p / q<1 .
$$

Dito isso, denotemos agora por $\mathrm{Q}$ o gerador de um processo de nascimento e morte em tempo contínuo em $\mathbb{N}$ com taxa de nascimento $p$ e taxa de morte $q, p<1 / 2$ e $q=1-p$, cuja distribuição

\footnotetext{
${ }^{1}$ Recordarmos que $\mathbf{P}_{\mathbf{w}}$ representa a lei do processo a partir de $\omega(0)=\mathbf{w}$ e $\mathbf{x}_{0}=\mathbf{0}$, como previamente estabelecido em $2.15, \mathbf{w} \in \Lambda$.
} 
invariante é $\nu$. Seja também $Q_{a}$ um gerador auxiliar dado por

$$
\mathrm{Q}_{a}=D \mathrm{Q}, \operatorname{com} D=\operatorname{diag}\left\{a^{n}\right\}_{n \in \mathbb{N}}, a>1,
$$

i.e., o gerador de um um processo de nascimento e morte com taxas de salto dadas por

$$
\mathrm{Q}_{a}(n, n+1)=p a^{n}, \quad \mathrm{Q}_{a}(n, n-1)=q a^{n}, \quad n \geqslant 1,
$$

e $\mathrm{Q}_{a}(0,1)=p$. Note que um processo de nascimento e morte com gerador $\mathrm{Q}_{a}$ é recorrente positivo e, portanto, não explosivo ([Norris, 1997], Capítulo 2, Teo 2.7.1(iii)). Denotaremos por $\nu_{a}$ a distribuição invariante associada a $\mathbf{Q}_{a}$, dada por

$$
\nu_{a}(k)=(1-\rho / a)(\rho / a)^{k}, \quad k \in \mathbb{N} .
$$

Lema 3.1. Seja $\mu$ uma distribuição de probabilidade em $\mathbb{N}$ com $\mu \leq \nu$. Então, para todo $t \in \mathbb{R}_{+}$,

$$
\mu e^{t Q} \leq \nu
$$

Demonstração. Sejam $\left(\mathrm{Y}_{t}\right)_{t \in \mathbb{R}_{+}}$e $\left(\mathrm{Y}_{t}^{\prime}\right)_{t \in \mathbb{R}_{+}}$dois processos de nascimentos e morte independentes com distribuições iniciais $\nu$ e $\mu$, respectivamente, ambos com gerador Q. Queremos provar que

$$
\mathbb{P}\left(\mathrm{Y}_{t} \geqslant k\right) \geqslant \mathbb{P}\left(\mathrm{Y}_{t}^{\prime} \geqslant k\right), \quad k \in \mathbb{N}, t \in \mathbb{R}_{+}
$$

Vamos verificar a validade de (3.6) a partir de um acoplamento clássico dos processos $\left(\mathrm{Y}_{t}\right)_{t \in \mathbb{R}_{+}}$e $\left(Y_{t}^{\prime}\right)_{t \in \mathbb{R}_{+}}$(vide [Thorisson, 2000], Capítulo 2, Seção 2, pg. 34) com as medidas iniciais acopladas via acoplamento quantílico (vide [Thorisson, 2000], Capítulo 1, Seção 3, pg. 3).

Por hipótese,

$$
\mathrm{F}(x):=\mathbb{P}\left(\mathrm{Y}_{0} \leqslant x\right) \leqslant \mathbb{P}\left(\mathrm{Y}_{0}^{\prime} \leqslant x\right)=: \mathrm{G}(x), \quad x \in \mathbb{R}_{+} .
$$

Denotemos por $\mathrm{F}^{-1}$ a inversa generalizada de $\mathrm{F}$, i.e.,

$$
\mathrm{F}^{-1}(u)=\inf \{x \in \mathbb{R}: \mathrm{F}(x) \geqslant u\}, u \in[0,1]
$$

De modo análogo, fica definida $\mathrm{G}^{-1}$. Observe que, de (3.7), por definição

$$
\mathrm{F}^{-1}(u) \geqslant \mathrm{G}^{-1}(u), \quad u \in[0,1]
$$

Seja $U$ uma uniforme padrão e consideremos $\left(\hat{\mathrm{Y}}_{t}\right)_{t \in \mathbb{R}_{+}}$e $\left(\hat{\mathrm{Y}}_{t}^{\prime}\right)_{t \in \mathbb{R}_{+}}$dois processos de nascimentos e morte com

$$
\hat{\mathrm{Y}}_{0}=\mathrm{F}^{-1}(U) \quad \text { e } \quad \hat{\mathrm{Y}}_{0}^{\prime}=\mathrm{G}^{-1}(U)
$$

e que, dadas essas condições iniciais, evoluem de modo independente, ambos com gerador Q. Com 
certo abuso de notação, denotemos também por $\mathbb{P}$ a lei conjunta de $\left(\hat{\mathrm{Y}}_{t}, \hat{\mathrm{Y}}_{t}^{\prime}\right)_{t \in \mathbb{R}_{+}}$. Note que

$$
\mathbb{P}\left(\hat{\mathrm{Y}}_{0} \leqslant x\right)=\mathbb{P}\left(\mathrm{F}^{-1}(U) \leqslant x\right)=\mathbb{P}(U \leqslant \mathrm{~F}(x))=\mathrm{F}(x),
$$

i.e. $\hat{\mathrm{Y}}_{0} \stackrel{d}{=} \mathrm{Y}_{0}$. De modo análogo, segue que $\hat{\mathrm{Y}}_{0}^{\prime} \stackrel{d}{=} \mathrm{Y}_{0}^{\prime}$. Além disso, de (3.9), $\hat{\mathrm{Y}}_{0} \geqslant \hat{\mathrm{Y}}_{0}^{\prime}$. Dado o gerador comum $Q$ a todos os processos previamente citados, isto é suficiente para concluir que $\left(\hat{\mathrm{Y}}_{t}, \hat{\mathrm{Y}}_{t}^{\prime}\right)_{t \in \mathbb{R}_{+}}$ é um acoplamento de $\left(\mathrm{Y}_{t}\right)_{t \in \mathbb{R}_{+}}$e $\left(\mathrm{Y}_{t}^{\prime}\right)_{t \in \mathbb{R}_{+}}$com $\hat{\mathrm{Y}}_{0} \geqslant \hat{\mathrm{Y}}_{0}^{\prime}$. Agora, definamos

$$
\mathrm{T}:=\inf \left\{s>0: \hat{\mathrm{Y}}_{t}=\hat{\mathrm{Y}}_{t}^{\prime}\right\}
$$

e consideremos $\left(\hat{\Upsilon}_{t}^{\prime \prime}\right)_{t \in \mathbb{R}_{+}}$definido por

$$
\hat{\mathrm{Y}}_{t}^{\prime \prime}= \begin{cases}\hat{\mathrm{Y}}_{t}^{\prime}, & \text { se } t<\mathrm{T} \\ \hat{\mathrm{Y}}_{t}, & \text { se } t>\mathrm{T},\end{cases}
$$

i.e., $\left(\hat{\mathbf{Y}}_{t}^{\prime \prime}\right)_{t \in \mathbb{R}_{+}}$segue a mesma trajetória de $\left(\hat{\mathrm{Y}}_{t}^{\prime}\right)_{t \in \mathbb{R}_{+}}$até o tempo $\mathrm{T}$ e a de $\left(\hat{\mathrm{Y}}_{t}\right)_{t \in \mathbb{R}_{+}}$após o tempo T. É fácil ver que $\left(\hat{Y}_{t}, \hat{Y}_{t}^{\prime}\right)_{t \in \mathbb{R}_{+}}$é um processo de Markov recorrente positivo em $\mathbb{N} \times \mathbb{N}$, donde segue, sem maiores dificuldades, a finitude quase certa de T. Além disso, como

$$
\{\mathrm{T}<t\}=\bigcup_{s \in \mathbb{Q}, s<t} \bigcup_{i \in \mathbb{N}}\left\{\left(\hat{\mathrm{Y}}_{s}, \hat{\mathrm{Y}}_{s}^{\prime}\right)=(i, i)\right\}
$$

T é um tempo de parada para $\left(\hat{\mathrm{Y}}_{t}, \hat{\mathrm{Y}}_{t}^{\prime}\right)_{t \in \mathbb{R}_{+}}$. Daí, pela propriedade forte de Markov, $\left(\hat{\mathrm{Y}}_{\mathrm{T}+s}\right)_{s \in \mathbb{R}_{+}}$e $\left(\hat{\mathrm{Y}}_{\mathrm{T}+s}^{\prime}\right)_{s \in \mathbb{R}_{+}}$são equivalentes em lei, uma vez que partem de um mesmo valor em $\mathrm{T}$ e evoluem de modo independente, ambos com o gerador Q. Assim, $\left(\hat{\mathrm{Y}}_{t}, \hat{\mathrm{Y}}_{t}^{\prime \prime}\right)_{t \in \mathbb{R}_{+}}$equivale a $\left(\hat{\mathrm{Y}}_{t}, \hat{\mathrm{Y}}_{t}^{\prime}\right)_{t \in \mathbb{R}_{+}}$, i.e., é um acoplamento de $\left(\mathrm{Y}_{t}\right)_{t \in \mathbb{R}_{+}}$e $\left(\mathrm{Y}_{t}^{\prime}\right)_{t \in \mathbb{R}_{+}}$. Além disso,

$$
\hat{\mathrm{Y}}_{t}=\hat{\mathrm{Y}}_{t}^{\prime \prime}
$$

para $t \geqslant \mathrm{~T}$ e

$$
\hat{Y}_{t}>\hat{Y}_{t}^{\prime \prime}
$$

para $t<\mathrm{T}$, uma vez que, sob o caso não trivial $\mathrm{T}>0, \hat{\mathrm{Y}}_{0}>\hat{\mathrm{Y}}_{0}^{\prime \prime}$ e, pela amplitude unitária dos saltos de um processo de nascimento e morte, as trajetórias de $\left(\hat{\mathrm{Y}}_{t}\right)_{t \in \mathbb{R}_{+}}$e $\left(\hat{\mathrm{Y}}_{t}^{\prime \prime}\right)_{t \in \mathbb{R}_{+}}$não podem se cruzar (isto ocorreria apenas se houvesse saltos simultâneos, o que é impossível: os tempos entre saltos dos processos são exponenciais independentes), apenas se encontrar e seguirem iguais a partir de então. Desse modo, para $k \in \mathbb{N}$,

$$
\mathbb{P}\left(\hat{\mathrm{Y}}_{t} \geqslant k\right) \geqslant \mathbb{P}\left(\hat{\mathrm{Y}}_{t}^{\prime \prime} \geqslant k\right), \quad t \in \mathbb{R}_{+}
$$

ou, de modo equivalente,

$$
\mathbb{P}\left(\mathrm{Y}_{t} \geqslant k\right) \geqslant \mathbb{P}\left(\mathrm{Y}_{t}^{\prime} \geqslant k\right), \quad k \in \mathbb{N}, t \in \mathbb{R}_{+} .
$$


A partir da relação expressa pela Equação (??), provaremos o seguinte Lema:

Lema 3.2. Seja $\mathrm{Q}_{a}$ como em (3.3), a $>1$. Então, para todo $t \in \mathbb{R}_{+}$,

$$
\nu e^{t Q_{a}}<\nu
$$

Demonstração. Seja $\left(\mathrm{Y}_{t}\right)_{t \in \mathbb{R}_{+}}$um processo estocástico em $\mathbb{N}$ com gerador $\mathrm{Q}_{a}$ e medida inicial $\nu$. Façamos $P_{n, j}(t):=\mathbb{P}\left(\mathrm{Y}_{t}=j \mid \mathrm{Y}_{0}=n\right), t$ real positivo, $n$ e $j$ naturais. Para $l \in \mathbb{N}$,

$$
\mathbb{P}\left(\mathrm{Y}_{t} \leqslant l\right)=\sum_{j \leqslant l} \mathbb{P}\left(\mathrm{Y}_{t}=j\right)=\sum_{j \leqslant l} \sum_{n \geqslant 0} \nu(n) P_{n, j}(t) .
$$

Pelo Teorema de Tonelli,

$$
\mathbb{P}\left(\mathrm{Y}_{t} \leqslant l\right)=\sum_{n \geqslant 0} \sum_{j \leqslant l} \nu(n) P_{n, j}(t) .
$$

As equações adiantadas de Kolmogorov para $\left(\mathrm{Y}_{t}\right)_{t \in \mathbb{R}_{+}}$são dadas por

$$
\begin{aligned}
& P_{n, 0}^{\prime}(t)=-p P_{n, 0}(t)+q a P_{n, 1}(t) \\
& P_{n, j}^{\prime}(t)=p a^{j-1} P_{n, j-1}(t)-a^{j} P_{n, j}(t)+q a^{j+1} P_{n, j+1}(t), \quad j \geqslant 1 ;
\end{aligned}
$$

para $n \geqslant 0$. Destas, obtemos que

$$
\left|\sum_{j \leqslant l} \nu(n) P_{n, j}^{\prime}(t)\right|=\nu(n)\left|P_{n, l+1}(t) q a^{l+1}-P_{n, l}(t) p a^{l}\right| \leqslant \nu(n) a^{l+1} .
$$

Uma vez que

$$
\nu(k)=(1-\rho) \rho^{k}, \quad k \in \mathbb{N}, \quad \rho=p / q<1,
$$

segue que $\sum_{n \in \mathbb{N}} \nu(n) a^{l+1}<\infty$ e, pelo Teste de Weierstrass (vide [Lima, 2010], Capítulo X, Teorema 2 , pg 370), decorre que $\sum_{n \geqslant 0} \sum_{j \leqslant l} \nu(n) P_{n, j}^{\prime}(t)$ é uniformemente convergente em $t$. Assim, por resultado bem conhecido (veja [Lima, 2010], Capitulo X, Corolário 2, pg 382), temos

$$
\mathbb{P}^{\prime}\left(\mathrm{Y}_{t} \leqslant l\right)=\sum_{n \geqslant 0} \sum_{j \leqslant l} \nu(n) P_{n, j}^{\prime}(t) .
$$

Agora, ressaltamos as equações atrasadas de Kolmogorov para $\left(\mathrm{Y}_{t}\right)_{t \in \mathbb{R}_{+}}$; dadas por

$$
\begin{aligned}
& P_{0, j}^{\prime}(t)=-p P_{0, j}(t)+p P_{1, j}(t) \\
& P_{n, j}^{\prime}(t)=q a^{n} P_{n-1, j}(t)-a^{n} P_{n, j}(t)+p a^{n} P_{n+1, j}(t), \quad n \geqslant 1,
\end{aligned}
$$

para $j \geqslant 0$. Substituindo estas em $(3.25)$ e tomando $d_{n}:=\mathbb{P}_{n}\left(\mathrm{Y}_{t} \leqslant l\right)-\mathbb{P}_{n+1}\left(\mathrm{Y}_{t} \leqslant l\right), n \in \mathbb{N}, b_{0}:=q$ e $b_{1}:=-p$, temos 


$$
\begin{aligned}
\mathbb{P}^{\prime}\left(\mathrm{Y}_{t} \leqslant l\right) & =\sum_{j \leqslant l} \nu(0) P_{0, j}^{\prime}(t)+\sum_{n \geqslant 1} \sum_{j \leqslant l} \nu(n) P_{n, j}^{\prime}(t) \\
& =-p(1-\rho) d_{0}+(1-\rho) \sum_{n \geqslant 1}(\rho a)^{n}\left(q d_{n-1}-p d_{n}\right) \\
& =-p(1-\rho) d_{0}+(1-\rho) \sum_{n \geqslant 1} \sum_{m \leqslant 1} b_{m}(\rho a)^{n} d_{n-1+m},
\end{aligned}
$$

com $\rho=p / q, m \in \mathbb{N}$. Vamos agora mostrar que a ordem das somas no segundo termo do lado direito de (3.28) pode ser alterada. Para tal, observe que

$$
\sum_{n \geqslant 1} \sum_{m \leqslant 1}\left|b_{m}\right|(\rho a)^{n} d_{n-1+m} \leqslant \sum_{n \geqslant 1}(\rho a)^{n}\left(d_{n-1} \vee d_{n}\right),
$$

$\operatorname{com} a \vee b=\max \{a, b\}$ e - tomando $\mathrm{H}_{n}:=\inf \left\{s>0: \mathrm{Y}_{s}=n\right\}, n \in \mathbb{N}$ - que, para $n \geqslant l$,

$$
\begin{aligned}
d_{n} & =\mathbb{P}_{n}\left(\mathrm{Y}_{t} \leqslant l\right)-\int_{0}^{t} \mathbb{P}_{n+1}\left(\mathrm{H}_{n} \in \mathrm{d} s\right) \mathbb{P}_{n}\left(\mathrm{Y}_{t-s} \leqslant l\right) \mathrm{d} s \\
& =\int_{0}^{t} \mathbb{P}_{n+1}\left(\mathrm{H}_{n} \in \mathrm{d} s\right)\left[\mathbb{P}_{n}\left(\mathrm{Y}_{t} \leqslant l\right)-\mathbb{P}_{n}\left(\mathrm{Y}_{t-s} \leqslant l\right)\right] \mathrm{d} s+\mathbb{P}_{n}\left(\mathrm{Y}_{t} \leqslant l\right) \int_{t}^{\infty} \mathbb{P}_{n+1}\left(\mathrm{H}_{n} \in \mathrm{d} s\right) \mathrm{d} s \\
& =\int_{0}^{t} \mathbb{P}_{n+1}\left(\mathrm{H}_{n} \in \mathrm{d} s\right)\left[\mathbb{P}_{n}\left(\mathrm{Y}_{t} \leqslant l, \mathrm{Y}_{t-s}>l\right)-\mathbb{P}_{n}\left(\mathrm{Y}_{t}>l, \mathrm{Y}_{t-s} \leqslant l\right)\right] \mathrm{d} s+\mathbb{P}_{n}\left(\mathrm{Y}_{t} \leqslant l\right) \mathbb{P}_{n+1}\left(\mathrm{H}_{n}>t\right) .
\end{aligned}
$$

Agora, dada $\left(V_{i}\right)_{i \in \mathbb{N}_{*}}$ uma sequência de exponenciais padrões independentes, seja $\left(\tilde{Y}_{k}\right)_{k \geqslant 0}$ a cadeia imersa de $\left(\mathrm{Y}_{t}\right)_{t \in \mathbb{R}_{+}}, \tilde{\mathrm{H}}_{n}=\inf \left\{k \geqslant 0: \tilde{\mathrm{Y}}_{k}=n\right\}$ e definamos uma variável auxiliar $\mathrm{H}_{n}^{\prime}=\sum_{i=1}^{\tilde{\mathrm{H}}_{n}} V_{i}$. Em palavras, $\tilde{\mathrm{H}}_{n}$ representa o instante em que a cadeia imersa $\left(\tilde{\mathrm{Y}}_{k}\right)_{k \geqslant 0}$ atinge o estado $n$ pela primeira vez e $\mathrm{H}_{n}^{\prime}$ o tempo necessário para um $\operatorname{PN} M(p, q)$ valer $n, n \in \mathbb{N}$. Note que, a partir de $\mathrm{Y}_{0}=n+1$, $\mathrm{H}_{n} \stackrel{s t}{\leq} \mathrm{H}_{n}^{\prime} / a^{n}$, donde segue pela Desigualdade de Markov que

$$
\mathbb{P}_{n+1}\left(\mathrm{H}_{n}>t\right) \leqslant \mathbb{P}_{n+1}\left(\mathrm{H}_{n}^{\prime}>a^{n} t\right) \leqslant\left[(q-p) t a^{n}\right]^{-1},
$$

uma vez que

$$
\mathbb{E}_{n+1}\left(\mathrm{H}_{n}^{\prime}\right)=\left.\frac{\mathrm{d}}{\mathrm{d} u} \mathbb{E}_{n+1}\left(e^{u \mathrm{H}_{n}^{\prime}}\right)\right|_{u=0}=\left.\frac{\mathrm{d}}{\mathrm{d} u}\left(\frac{1-u-\sqrt{(1-u)^{2}-4 p q}}{2 p}\right)\right|_{u=0}=\frac{1}{q-p}
$$

Além disso, por $\left(\mathrm{Y}_{t}\right)_{t \in \mathbb{R}_{+}}$ser um processo de Markov,

$$
\begin{aligned}
\mathbb{P}_{n}\left(\mathrm{Y}_{t} \leqslant l, \mathrm{Y}_{t-s}>l\right) & =\sum_{j \geqslant l+1} \mathbb{P}_{n}\left(\mathrm{Y}_{t-s}=j\right) \mathbb{P}_{j}\left(\mathrm{Y}_{s} \leqslant l\right) \\
& \leqslant \sum_{j \geqslant l+1} \mathbb{P}_{n}\left(\mathrm{Y}_{t-s}=j\right) \mathbb{P}_{l+1}\left(\mathrm{Y}_{s} \leqslant l\right) \\
& \leqslant \sum_{j \geqslant l+1} \mathbb{P}_{n}\left(\mathrm{Y}_{t-s}=j\right)\left(1-e^{-a^{l+1} s}\right) \leqslant 1-e^{-a^{l+1} s} .
\end{aligned}
$$


Assim,

$$
\begin{aligned}
d_{n} & \leqslant \int_{0}^{t} \mathbb{P}_{n+1}\left(\mathrm{H}_{n} \in \mathrm{d} s\right)\left(1-e^{-a^{l+1} s}\right) \mathrm{d} s+\left[(q-p) t a^{n}\right]^{-1}, \\
& \leqslant \int_{0}^{\infty} \mathbb{P}_{n+1}\left(\mathrm{H}_{n} \in \mathrm{d} s\right)\left(1-e^{-a^{l+1} s}\right) \mathrm{d} s+\left[(q-p) t a^{n}\right]^{-1} \\
& =\mathbb{E}_{n+1}\left(1-e^{-a^{l+1} \mathrm{H}_{n}}\right)+\left[(q-p) t a^{n}\right]^{-1} \\
& \leqslant \mathbb{E}_{n+1}\left(\left[\frac{1-e^{-a^{l+1} \mathrm{H}_{n}^{\prime} / a^{n}}}{a^{l+1} \mathrm{H}_{n}^{\prime} / a^{n}}\right]\left[a^{l+1} \mathrm{H}_{n}^{\prime} / a^{n}\right]\right)+\left[(q-p) t a^{n}\right]^{-1} \\
& \leqslant \mathbb{E}_{n+1}\left(\frac{a^{l+1} \mathrm{H}_{n}^{\prime}}{a^{n}}\right)+\frac{1}{(q-p) t a^{n}} \leqslant \frac{t a^{l+1}+1}{(q-p) t a^{n}} .
\end{aligned}
$$

Logo

$$
\sum_{n \geqslant l} \sum_{m \leqslant 1}\left|b_{m}\right|(\rho a)^{n} d_{n-1+m} \leqslant \sum_{n \geqslant l}(\rho a)^{n}\left[\frac{t a^{l+1}+1}{(q-p) t a^{n-1}}\right]=\left[\frac{q a\left(t a^{l+1}+1\right)}{t(q-p)^{2}}\right] \rho^{l+1}<\infty,
$$

isto é, a série dada em (3.29) converge. Daí, por Fubini, (3.28) pode ser expressa como

$$
\begin{aligned}
\mathbb{P}^{\prime}\left(Y_{t} \leqslant l\right) & =(1-\rho) \sum_{m \leqslant 1} \sum_{n \geqslant 1} b_{m}(\rho a)^{n} d_{n-1+m}-p(1-\rho) d_{0} \\
& =(1-\rho) \sum_{n \geqslant 1} b_{0}(\rho a)^{n} d_{n-1}+(1-\rho) \sum_{n \geqslant 1} b_{1}(\rho a)^{n} d_{n}-p(1-\rho) d_{0} \\
& =(1-\rho) \sum_{n \geqslant 1} b_{0}(\rho a)^{n+1} d_{n}+(1-\rho) \sum_{n \geqslant 1} b_{1}(\rho a)^{n} d_{n}+d_{0}(1-\rho)(p a-p) \\
& =(1-\rho) \sum_{n \geqslant 1}(\rho a)^{n} d_{n}(p a-p)+d_{0}(1-\rho)(p a-p)>0 .
\end{aligned}
$$

Daí, para $l \in \mathbb{N}$,

$$
\mathbb{P}^{\prime}\left(\mathrm{Y}_{t} \leqslant l\right)>0
$$

Como

$$
\mathbb{P}\left(\mathrm{Y}_{t}>l\right)=1-\mathbb{P}\left(\mathrm{Y}_{t} \leqslant l\right),
$$

temos

$$
\mathbb{P}^{\prime}\left(\mathrm{Y}_{t}>l\right)=-\mathbb{P}^{\prime}\left(\mathrm{Y}_{t} \leqslant l\right)<0
$$

o que conclui a prova.

Corolário 3.1. Suponha $\hat{\mu}_{0} \leq \hat{\nu}$. Então $\hat{\mu}_{\tau_{n}}<\hat{\nu}, n \in \mathbb{N}_{*}$.

Demonstração. Para $k \in \mathbb{N}_{*}$, seja $f_{k}=\left(f_{k}(n)\right)_{n \in \mathbb{N}}, \operatorname{com} f_{k}(n)=\mathbb{1}\{n \geqslant k\}, n \in \mathbb{N}$. Para $\mathbf{x} \neq \mathbf{0}$,

$$
\mu_{\mathbf{x}, 0} e^{\Delta_{1} \mathbf{Q}} f_{k}=\int_{0}^{\infty} \mathbf{P}_{\hat{\mu}_{0}}\left(\omega_{\mathbf{x}}(s) \geqslant k\right) \mathbf{P}_{\hat{\mu}_{0}}\left(\Delta_{1} \in d s\right) d s \leqslant \int_{0}^{\infty} \nu f_{k} \mathbf{P}_{\hat{\mu}_{0}}\left(\Delta_{1} \in d s\right) d s=\nu f_{k},
$$

com a primeira e segunda passagens decorrentes, respectivamente, da independência entre $\Delta_{1} \mathrm{e}$ $\omega_{\mathbf{x}}, \mathbf{x} \neq \mathbf{0}$, e da dominação estocástica vista no Lema 3.1. Para $\mathbf{x}=\mathbf{0}$, vamos a uma descrição alternativa de $\omega_{0}$ : 
Sejam $\left(V_{n}\right)_{n \in \mathbb{N}_{*}}$ exponenciais padrões independentes e, independente destas, $(\mathrm{w}(n))_{n \in \mathbb{N}}$ um cadeia de nascimento e morte em $\mathbb{N}$ com probabilidade de nascimento $p \in(0,1 / 2)$ e probabilidade de morte $q=1-p>p$, com distribuição inicial $\mu_{\mathbf{0}, 0}$. Façamos $T_{n}=\sum_{k=1}^{n} V_{k}, n \in \mathbb{N}$. Então

$$
\omega_{\mathbf{0}}(t)=\sum_{k=0}^{\infty} \mathrm{w}(k) \mathbb{1}\left\{T_{k} \leqslant t<T_{k+1}\right\} .
$$

Nesses termos, é fácil ver que, para $t \in\left(T_{n}, T_{n+1}\right)$,

$$
I_{\mathbf{0}}(t)=\sum_{k=1}^{n} a^{-\mathrm{w}(k-1)} V_{k}+a^{-\mathrm{w}(n)}\left(t-T_{n}\right)
$$

Agora, façamos, para $n \in \mathbb{N}_{*}, \hat{V}_{n}=a^{-\mathrm{w}(n-1)} V_{n}$ e definamos

$$
\hat{\omega}_{\mathbf{0}}(t)=\sum_{k=0}^{\infty} \mathrm{w}(k) \mathbb{1}\left\{\hat{T}_{k} \leqslant t<\hat{T}_{k+1}\right\},
$$

em que $\hat{T}_{n}=\sum_{k=1}^{n} \hat{V}_{k}$. Da definição, $\omega_{\mathbf{0}}(t)$ e $\hat{\omega}_{\mathbf{0}}(t)$ têm a mesma cadeia imersa. As diferenças consistem de contrações nos tempos de permanência em cada estado. De fato, $\hat{\omega}_{\mathbf{0}}(t)$ é um processo de nascimento e morte com distribuição inicial $\mu_{\mathbf{0}, 0}$ e gerador $Q_{a}$, com $Q_{a}$ definido em (3.42). Além disso, por construção, $\omega_{\mathbf{0}}$ em $\Delta_{1}$ equivale em distribuição a $\hat{\omega}_{\mathbf{0}}$ em $I_{0}\left(\Delta_{1}\right)$, com $I_{0}\left(\Delta_{1}\right)$, pelo Lema 2.1, uma exponencial padrão independente de $\omega$. Para tornar mais claro, veja a Figura 3.1

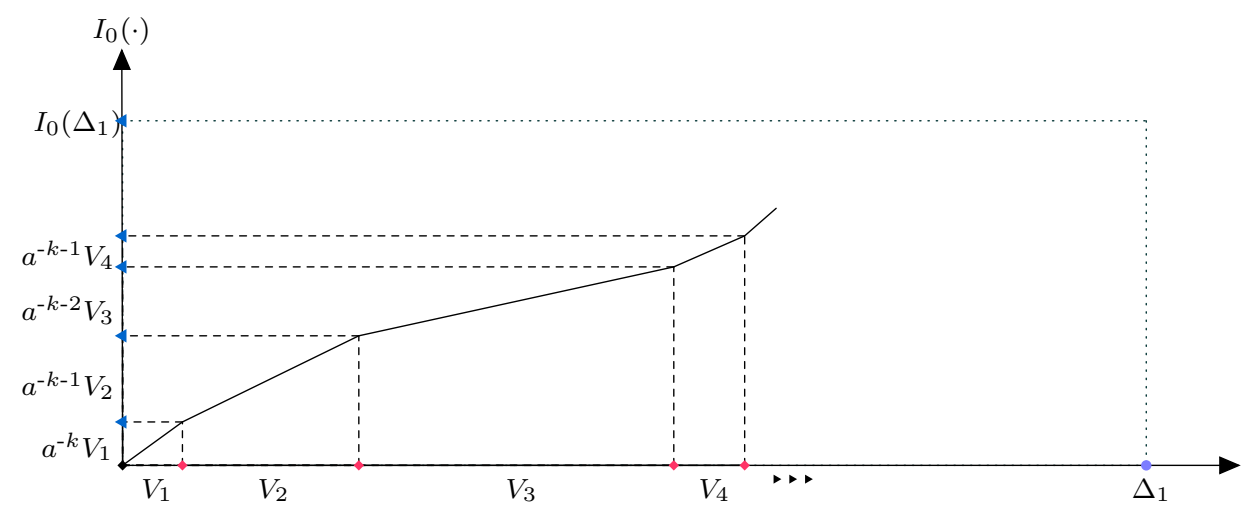

Figura 3.1: $I_{0}(\cdot)$ sob uma possivel realização de $\omega_{\mathbf{0}} \operatorname{com} \omega_{\mathbf{0}, 0} \equiv k, k \in \mathbb{N}$.

Assim, temos que

$$
\begin{aligned}
\mu_{\mathbf{0}} e^{\Delta_{1} Q} f_{k}=\mu_{\mathbf{0}} e^{I\left(\Delta_{1}\right) Q_{a}} f_{k} & =\int_{0}^{\infty} \mathbf{P}_{\hat{\mu}_{0}}\left(\hat{\omega}_{\mathbf{0}}\left(I\left(\Delta_{1}\right)\right) \geqslant k \mid I\left(\Delta_{1}\right)=s\right) e^{-s} d s \\
& =\int_{0}^{\infty} \mathbf{P}_{\hat{\mu}_{0}}\left(\hat{\omega}_{\mathbf{0}}(s) \geqslant k\right) e^{-s} d s \\
& \leqslant \int_{0}^{\infty} \mathbf{P}_{\hat{\nu}}\left(\hat{\omega}_{\mathbf{0}}(s) \geqslant k\right) e^{-s} d s<\int_{0}^{\infty} \nu f_{k} e^{-s} d s=\nu f_{k}
\end{aligned}
$$

com a quinta e quarta passagens justificadas pelo Lema 3.2 e uma extensão direta do Lema 3.1(os argumentos lá permanecem válidos ao considerar o gerador $Q_{a}$ em vez de $Q$ ), respectivamente. Com 
isso, verificamos que

$$
\hat{\mu}_{\tau_{1}}=\bigotimes_{\mathbf{x} \in \mathbb{Z}^{d}} \mu_{\mathbf{x}, \Delta_{1}} \prec \underset{\mathbf{x} \in \mathbb{Z}^{d}}{\bigotimes} \nu=\hat{\nu}
$$

Vimos que vale para $i=1$. Suponhamos válida para $i=n$. Para verificar que vale para $i=n+1$, basta iterar o argumento visto para o caso $i=1$ a partir do processo

$$
(\tilde{X}(s), \tilde{\omega}(s))_{s \in \mathbb{R}_{+}}:=\left(X\left(\tau_{n}+s\right), \omega\left(\tau_{n}+s\right)\right)_{s \in \mathbb{R}_{+}},
$$

que equivale, pela Propriedade Forte de Markov, ao processo em estudo sob $\mathbf{P}_{\hat{\mu}_{\tau_{n}}, \mathrm{x}_{n}}$ com $\hat{\mu}_{\tau_{n}}$, por hipótese, dominada estocasticamente por $\hat{\nu}$. Pelo princípio da indução, fica verificado o resultado.

Diremos que $\mathrm{Q}_{a}$ é o gerador associado a $\omega_{\mathrm{x}_{n}}$, em $t \in\left[\tau_{n}, \tau_{n+1}\right), n \in \mathbb{N}$, ou ainda, em palavras, o gerador associado ao ambiente na posição da partícula.

Corolário 3.2. Suponha $\hat{\mu}_{0} \leq \hat{\nu}$. Então, para $n \in \mathbb{N}_{*}$,

$$
\mathbf{E}_{\hat{\mu}_{0}}\left(\Delta_{n}\right) \leqslant \mathbf{E}_{\hat{\nu}}\left(\Delta_{1}\right)
$$

Demonstração. Vamos verificar para $n=1$. Sejam $\left(\mathrm{Y}_{t}\right)_{t \in \mathbb{R}_{+}} \sim P N M(p, q)$ e $\left(\mathrm{Y}_{t}^{\prime}\right)_{t \in \mathbb{R}_{+}} \sim P N M(p, q)$ independentes, $p \in(0,1 / 2), q=1-p$, com distribuições iniciais $\nu$ e $\mu_{0,0}$, respectivamente. Agora, seja $\left(\hat{Y}_{t}, \hat{Y}_{t}^{\prime \prime}\right)_{t \in \mathbb{R}_{+}}$um acoplamento de $\left(\mathrm{Y}_{t}\right)_{t \in \mathbb{R}_{+}}$e $\left(\mathrm{Y}_{t}^{\prime}\right)_{t \in \mathbb{R}_{+}}$, definido como na demonstração do Lema 3.1. Como $\hat{Y}_{u} \geqslant \hat{Y}_{u}^{\prime \prime}$ para todo $u \in \mathbb{R}_{+}$, é claro que, para $t \in \mathbb{R}_{+}$,

$$
\exp \left\{-\int_{0}^{t} a^{-\hat{Y}_{u}} d u\right\} \geqslant \exp \left\{-\int_{0}^{t} a^{-\hat{Y}_{u}^{\prime \prime}} d u\right\}
$$

Daí, para cada $t \in \mathbb{R}_{+}$,

$$
\mathbb{P}\left(\exp \left\{-\int_{0}^{t} a^{-\hat{Y}_{u}} d u\right\}>s\right) \geqslant \mathbb{P}\left(\exp \left\{-\int_{0}^{t} a^{-\hat{Y}_{u}^{\prime \prime}} d u\right\}>s\right), \quad s \in \mathbb{R}_{+},
$$

e, portanto, integrando em $s$ em ambos os lados,

$$
\mathbb{E}\left(\exp \left\{-\int_{0}^{t} a^{-\hat{Y}_{u}} d u\right\}\right) \geqslant \mathbb{E}\left(\exp \left\{-\int_{0}^{t} a^{-\hat{Y}_{u}^{\prime \prime}} d u\right\}\right)
$$

Agora, basta ver que

$$
\begin{aligned}
\mathbf{E}_{\hat{\nu}}\left(\Delta_{1}\right)=\int_{0}^{\infty} \mathbf{P}_{\hat{\nu}}\left(\Delta_{1}^{k}>s\right) d s & =\int_{0}^{\infty} \mathrm{E}_{\hat{\nu}}\left(e^{-I_{\mathbf{0}}(t)}\right) d t \\
& =\int_{0}^{\infty} \mathbb{E}\left(\exp \left\{-\int_{0}^{t} a^{-\hat{\Upsilon}_{u}} d u\right\}\right) d t \\
& \geqslant \int_{0}^{\infty} \mathbb{E}\left(\exp \left\{-\int_{0}^{t} a^{-\hat{\Upsilon}_{u}^{\prime \prime}} d u\right\}\right) d t \\
& =\int_{0}^{\infty} \mathrm{E}_{\hat{\mu}_{0}}\left(e^{-I_{\mathbf{0}}(t)}\right) d t=\int_{0}^{\infty} \mathbf{P}_{\hat{\mu}_{0}}\left(\Delta_{1}>s\right) d s=\mathbf{E}_{\hat{\mu}_{0}}\left(\Delta_{1}\right) .
\end{aligned}
$$


Para $n \geqslant 2$, observe que $\Delta_{n}$, sob $\mathbf{P}_{\hat{\mu}_{0}}$, equivale, pela propriedade forte de Markov, a $\tilde{\Delta}_{1}$, $\operatorname{com} \tilde{\Delta}_{1}$ o tempo até o primeiro salto de $(\tilde{X}(t))_{t \in \mathbb{R}_{+}}$, com

$$
(\tilde{X}(s), \tilde{\omega}(s))_{s \in \mathbb{R}_{+}}:=\left(X\left(\tau_{n}+s\right), \omega\left(\tau_{n}+s\right)\right)_{s \in \mathbb{R}_{+}} .
$$

Além disso, do Corolário 3.1, $\hat{\mu}_{\tau_{n}} \leq \hat{\nu}$. Assim,

$$
\mathbf{E}_{\hat{\mu}_{0}}\left(\Delta_{n+1}\right)=\mathbf{E}_{\hat{\mu}_{0}}\left(\tilde{\Delta}_{1}\right)=\mathbf{E}_{\hat{\mu}_{\tau n}}\left(\Delta_{1}\right) \leqslant \mathbf{E}_{\hat{\nu}}\left(\Delta_{1}\right)
$$

Proposição 3.1. Existe $\mu \in \mathbb{R}_{+}$tal que

$$
\frac{\tau_{n}}{n} \rightarrow \mu \quad \mathbf{P}_{\mathbf{0}}-\text { q.c. } \quad \text { quando } n \nearrow+\infty .
$$

Demonstração. Vamos dividir a prova em duas partes. Na Parte a, definimos um arranjo superaditivo de variáveis aleatórias $\left\{\mathrm{L}_{m, n}: m, n \in \mathbb{N}, m \leqslant n\right\}$ tal que $\mathrm{L}_{0, n}$ equivale a $\tau_{n}$ sob $\mathbf{P}_{\mathbf{0}}$. Na Parte b, mostramos que $\left\{-\mathrm{L}_{m, n}: m, n \in \mathbb{N}, m \leqslant n\right\}$ satisfaz as condições para o uso do Teorema Ergódico Subaditivo (veja [Durret, 2010], Capitulo 7, Seção 4, Teorema 7.4.1), de onde segue o resultado.

- Parte a: Seja $(X(t))_{t \in \mathbb{R}_{+}}$uma versão do passeio em meio aleatório em estudo sob $\mathbf{P}_{\mathbf{0}}$. Por $\left(\tau_{n}\right)_{n \in \mathbb{N}}$ indicamos, como usual, os respectivos tempos de salto. Ressaltamos também os elementos aleatórios, conforme Construção II do modelo, para a obtenção de $(X(t))_{t \in \mathbb{R}_{+}}$:

1. Meio: $\omega=\left\{\omega_{\mathbf{x}}: \mathbf{x} \in \mathbb{Z}^{d}\right\} \operatorname{com} \omega(0)=\mathbf{0}$;

2. Dinâmica:

2.1. $\mathrm{V}:=\left\{\mathrm{V}_{i}: i \in \mathbb{N}_{*}\right\}$ exponenciais padrões independentes entre si e de $\omega$; e

2.2. $\xi:=\left\{\xi_{i}: i \in \mathbb{N}_{*}\right\}$ vetores aleatórios i.i.d. com distribuição $\pi$ independentes de $\omega$ e $\mathrm{V}$.

Agora, para $s \in \mathbb{R}_{+}$fixado, dados $(X(t), \omega(t))_{t \in[0, s]},(X(s), \omega(s))=:\left(\times(s),\left(n_{\mathbf{x}}\right)_{\mathbf{x} \in \mathbb{Z}^{d}}\right)$, definamos uma versão do meio

$$
\omega^{s}(\cdot)=\left\{\omega_{\mathbf{x}}^{s}(\cdot): \mathbf{x} \in \mathbb{Z}^{d}\right\}
$$

em que $\left(\omega_{\mathbf{x}}(s+t), \omega_{\mathbf{x}}^{s}(t)\right)_{t \in \mathbb{R}_{+}}$consitem de um acoplamento clássico de dois $\operatorname{PNM}(p, q)^{\prime} s$ independentes com $\omega_{\mathbf{x}}(s)=n_{\mathbf{x}}$ e $\omega_{\mathbf{x}}^{s}(0) \equiv 0, n_{\mathbf{x}} \in \mathbb{N}, \times(s)$ e $\mathbf{x}$ em $\mathbb{Z}^{d}$ (vide [Thorisson, 2000], Capítulo 2, Seção 2, pg. 34). Segue que $\omega^{s}$ e $\omega$ equivalem em distrubuição e que, das propriedades do acoplamento clássico de $P N M^{\prime} s$ já ressaltadas na demostração do Lema 3.1,

$$
\omega_{\mathbf{x}}(s+t) \geqslant \omega_{\mathbf{x}}^{s}(t), \quad \mathbf{x} \in \mathbb{Z}^{d}, t \in \mathbb{R}_{+} .
$$

Denotemos agora por $\mathrm{n}_{s}$ o número de saltos de $(X(t))_{t \in \mathbb{R}_{+}}$observado até o tempo $s$ e definamos o processo $\left(X^{s}(t)\right)_{t \in \mathbb{R}_{+}}$dado por

$$
X^{s}(t)= \begin{cases}X(t), & t<s \\ Y^{s}(t-s), & t \geqslant s\end{cases}
$$


em que $\left(Y^{s}(t)\right)_{t \in \mathbb{R}_{+}}$é uma versão do passeio aleatório em estudo sob o meio $\omega^{s}$ a partir de $Y^{s}(0)=x(s)$ cuja dinâmica é construída, tendo em vista a Construção II do processo, com os objetos $\left\{\xi_{i}: i \in \mathbb{N}_{*}, i \geqslant \mathrm{n}_{s}\right\}$ e $\left\{\mathrm{V}_{i}: i \in \mathbb{N}_{*}, i \geqslant \mathrm{n}_{s}\right\}$, isto é, a cadeia imersa de $\left(Y^{s}(t)\right)_{t \in \mathbb{R}_{+}}-$ $\operatorname{digamos}\left(\mathrm{y}_{n}^{s}\right)_{n \in \mathbb{N}}$ - fica definida por

$$
\mathrm{y}_{n}^{s}=\mathrm{x}(s)+\sum_{i=1}^{n} \xi_{\mathrm{n}_{s}+i}
$$

e os tempos de salto de $\left(Y^{s}(t)\right)_{t \in \mathbb{R}_{+}}$- denotemo-os por $\left(\sigma_{n}^{s}\right)_{n \in \mathbb{N}}$ - ficam dados, de modo recursivo, por $\sigma_{0}^{s}=0$ e

$$
\sigma_{n}^{s}=\sigma_{n-1}^{s}+\left(I_{n}^{s}\right)^{-1}\left(V_{\mathrm{n}_{s}+n}\right), \quad n \in \mathbb{N}_{*},
$$

em que, dado $\sigma_{n}^{s}$,

$$
I_{n+1}^{s}(t)=\int_{\sigma_{n}^{s}}^{\sigma_{n}^{s}+t} a^{-\omega_{y_{n}^{s}}^{s}(r)} d r, \quad n \in \mathbb{N}
$$

Por fim, para $m, n$ naturais, $m \leqslant n$, definimos $\mathrm{L}_{m, n}=\sigma_{n-m}^{\tau_{m}}$, i.e., o tempo para que $\left(Y^{\tau_{m}}(t)\right)_{t \in \mathbb{R}_{+}}$ performe $n-m$ saltos ou, de modo equivalente, o tempo entre o $m$-ésimo e $n$-ésimo saltos de $\left(X^{\tau_{m}}(t)\right)_{t \in \mathbb{R}_{+}}$. Da construção, é claro que $\left(X^{\tau_{0}}(t)\right)_{t \in \mathbb{R}_{+}}$é o mesmo que $(X(t))_{t \in \mathbb{R}_{+}}$, de onde segue que $\mathrm{L}_{0, n}=\tau_{n}$.

- Parte b: Vamos verificar que

b.1) $\mathrm{L}_{0, n} \geqslant \mathrm{~L}_{0, m}+\mathrm{L}_{m, n}$

b.2) $\left\{\mathrm{L}_{n k,(n+1) k}, n \in \mathbb{N}\right\}$ é ergódica para cada $k \in \mathbb{N}$;

b.3) A distribuição de $\left\{\mathrm{L}_{n, n+k}: k \geqslant 1\right\}$ não depende de $n \in \mathbb{N}$;

b.4) Existe $\gamma_{0}<+\infty$ tal que $\mathbf{E}\left(\mathrm{L}_{0, n}\right) \leqslant \gamma_{0} n$.

Feito isso, o resultado segue de uma aplicação do Teorema Ergódico Subaditivo $-\mathrm{L}_{m, n}$, (veja [Durret, 2010], Capitulo 7, Seção 4, Teorema 7.4.1).

b.1) Primeiramente, observe que, para cada $m \in \mathbb{N}$,

$$
\mathrm{L}_{0, n}=\mathrm{L}_{0, m}+\tau_{n}-\tau_{m}, \quad n \in \mathbb{N}, n \geqslant m .
$$

Assim, basta provar que

$$
\Delta_{m, n}:=\tau_{n}-\tau_{m} \geqslant \mathrm{~L}_{m, n},
$$

o que faremos por indução. Para $n=m$, a igualdade é trivial. Para $n=m+1$, temos que

$$
\Delta_{m, m+1}=I_{m}^{-1}\left(V_{m+1}\right)
$$

com

$$
I_{m}(t)=\int_{\tau_{m}}^{\tau_{m}+t} a^{-\omega_{\mathrm{x}_{m}}(r)} d r,
$$

$\left(\mathrm{x}_{k}\right)_{k \in \mathbb{N}}$ a cadeia imersa de $(X(t))_{t \in \mathbb{R}_{+}}$; e

$$
\mathrm{L}_{m, n}=\left(I_{1}^{m}\right)^{-1}\left(V_{m+1}\right)
$$


$\operatorname{com} I_{k}^{m}:=I_{k}^{\tau_{m}}, k \in \mathbb{N}$, i.e.,

$$
I_{1}^{m}(t)=\int_{0}^{t} a^{-\omega_{x}^{\tau_{m}}(r)} d r
$$

Da Desigualdade (3.54) é direto que

$$
I_{m}(t) \leqslant I_{1}^{m}(t)
$$

de onde segue que $I_{m}^{-1}(t) \geqslant\left(I_{1}^{m}\right)^{-1}(t), t \in \mathbb{R}_{+}$, e, portanto, a validade de (3.60) para $n=m+1$. Suponhamos que vale para $n=m+k, k \in \mathbb{N}_{*}$. Então, ou

$$
\Delta_{m, m+k} \geqslant \mathrm{~L}_{m, m+k+1} \quad \Longrightarrow \quad \Delta_{m, m+k+1} \geqslant \mathrm{~L}_{m, m+k+1},
$$

$\mathrm{Ou}$

$$
\Delta_{m, m+k}<\mathrm{L}_{m, m+k+1} .
$$

Mas nesse segundo caso, também é impossível $\Delta_{m, m+k+1}<\mathrm{L}_{m, m+k+1}$. De fato, da hipótese de indução, segue que

$$
\Delta_{m, m+k+1}=\Delta_{m, m+k}+\Delta_{m+k, m+k+1} \geqslant \mathrm{~L}_{m, m+k}+\Delta_{m+k, m+k+1} .
$$

Para concluir, vamos provar, por contradição, que é impossível que

$$
\Delta_{m+k, m+k+1}<\mathrm{L}_{m, m+k+1}-\mathrm{L}_{m, m+k} .
$$

Suponhamos que a afirmação acima seja verdadeira. Por construção, temos que

$$
V_{m+k+1}=I_{m+k}\left(\Delta_{m+k, m+k+1}\right)=I_{k}^{m}\left(\mathrm{~L}_{m \cdot m+k+1}-\mathrm{L}_{m \cdot m+k}\right)
$$

Assim, sob a validade de $\Delta_{m+k, m+k+1}<\mathrm{L}_{m, m+k+1}-\mathrm{L}_{m, m+k}$,

$$
\begin{aligned}
\mathrm{V}_{m+k+1}=I_{m+k}\left(\tau_{m+k+1}-\tau_{m+k}\right) & =\int_{\tau_{m+k}}^{\tau_{m+k+1}} a^{-\omega_{x_{m+k}}(s)} d s \\
& =\int_{0}^{\Delta_{m+k, m+k+1}} a^{-\omega_{x_{m+k}}\left(\tau_{m+k}+r\right)} d r \\
& <\int_{0}^{\mathrm{L}_{m, m+k+1}-\mathrm{L}_{m, m+k}} a^{-\omega_{x_{n}}^{\tau_{m+k}}(r)} d r \\
& =I_{k}^{m}\left(\mathrm{~L}_{m . m+k+1}-\mathrm{L}_{m . m+k}\right)=\mathrm{V}_{m+k+1},
\end{aligned}
$$

o que é um absurdo. Pelo princípio da indução, fica verificado o item b.1).

b.2) Basta ver que $\left\{\mathrm{L}_{n k,(n+1) k}: n \in \mathbb{N}\right\}$ é um processo definido por variáveis aleatórias i.i.d. e, portanto, ergódico. De fato, para qualquer $J \subset\{1, \ldots, k\}$ e $t_{i} \in \mathbb{R}_{+}, i \in J$,

$$
\mathbf{P}_{\mathbf{0}}\left(\mathrm{L}_{n k,(n+1) k}<t \mid \mathrm{L}_{n i,(n+1) i}=t_{i}, i \in I\right)=\mathbf{P}_{\mathbf{0}}\left(\tau_{k}<t\right), \quad t \in \mathbb{R}_{+} .
$$

b.3) Por construção, $\left(\mathrm{L}_{n, n+k}\right)_{k \in \mathbb{N}_{*}}$ equivale em distribuição a $\left(\tau_{k}\right)_{k \in \mathbb{N}_{*}}$, cuja distribuição não 
depende de $n$, qualquer que seja $n \in \mathbb{N}$.

b.4) Do Corolário 3.2, temos que

$$
\mathbf{E}\left(\mathrm{L}_{0, n}\right)=\mathbf{E}_{\mathbf{0}}\left(\tau_{n}\right)=\sum_{k=1}^{n} \mathbf{E}_{\mathbf{0}}\left(\Delta_{k}\right) \leqslant n \mathbf{E}_{\hat{\nu}}\left(\tau_{1}\right)
$$

Logo, para findar a prova, vamos mostrar que $\mathbf{E}_{\hat{\nu}}\left(\tau_{1}\right)<\infty$. Temos

$$
\begin{aligned}
\mathbf{E}_{\hat{\nu}}\left(\Delta_{1}\right) & =\int_{0}^{\infty} \mathbf{P}_{\hat{\nu}}\left(\Delta_{1}>t\right) d t \\
& =\int_{0}^{\infty} \mathrm{E}_{\hat{\nu}}\left(e^{-I_{0}(t)}\right) d t \\
& =\int_{0}^{+\infty} \mathrm{E}_{\hat{\nu}}\left(e^{-I_{0}(t)} ; I_{0}(t) \geqslant \epsilon t\right) d t+\int_{0}^{+\infty} \mathrm{E}_{\hat{\nu}}\left(e^{-I_{0}(t)} ; I_{0}(t)<\epsilon t\right) d t \\
& \leqslant \epsilon^{-1}+\int_{0}^{+\infty} \mathbf{P}_{\hat{\nu}}\left(I_{0}(t)<\epsilon t\right) d t \\
& \leqslant \epsilon^{-1}+\int_{0}^{+\infty} \mathbf{P}_{\hat{\nu}}\left(\int_{0}^{t} \mathbb{1}\left\{\omega_{0}(s)=0\right\} d s<\epsilon t\right) d t .
\end{aligned}
$$

Para $k \in \mathbb{N}$, façamos $\mathbf{k}=k \times \mathbf{1}$. Condicionando no estado inicial do ambiente na origem, para cada $\delta>0$ e para cada $t \in \mathbb{R}_{+}$,

$$
\begin{aligned}
\mathbf{P}_{\hat{\nu}}\left(\int_{0}^{t} \mathbb{1}\left\{\omega_{\mathbf{0}}(s)=0\right\} d s<\epsilon t\right)= & \sum_{k=1}^{\mid \delta t\rfloor} \mathbf{P}_{\mathbf{k}}\left(\int_{0}^{t} \mathbb{1}\left\{\omega_{\mathbf{0}}(s)=0\right\} d s<\epsilon t\right)(1-\rho) \rho^{k}+ \\
& +\sum_{k=\lceil\delta t \mid}^{\infty} \mathbf{P}_{\mathbf{k}}\left(\int_{0}^{t} \mathbb{1}\left\{\omega_{\mathbf{0}}(s)=0\right\} d s<\epsilon t\right)(1-\rho) \rho^{k} \\
\leqslant & \sum_{k=1}^{|\delta t|} \mathbf{P}_{\mathbf{k}}\left(\int_{0}^{t} \mathbb{1}\left\{\omega_{\mathbf{0}}(s)=0\right\} d s<\epsilon t\right)+\sum_{k=\lceil\delta t]}^{\infty}(1-\rho) \rho^{k} \\
\leqslant & \sum_{k=1}^{\mid \delta t\rfloor} \mathbf{P}_{\mathbf{k}}\left(\int_{0}^{t} \mathbb{1}\left\{\omega_{\mathbf{0}}(s)=0\right\} d s<\epsilon t\right)+e^{t \delta \ln \rho} .
\end{aligned}
$$

Assim,

$$
\mathbf{E}_{\hat{\nu}}\left(\tau_{1}\right) \leqslant \epsilon^{-1}+\frac{1}{\delta|\log \rho|}+\int_{0}^{+\infty} \sum_{k=1}^{|\delta t|} \mathbf{P}_{\mathbf{k}}\left(\int_{0}^{t} \mathbb{1}\left\{\omega_{\mathbf{0}}(s)=0\right\} d s<\epsilon t\right) d t .
$$

Agora, façamos $W_{0}=\inf \left\{s>0: \omega_{0}(s)=0\right\}$ e definamos

$$
\begin{gathered}
Z_{1}=\inf \left\{s>W_{0}: \omega_{\mathbf{0}}(s) \neq 0\right\}-W_{0}, \\
W_{1}=\inf \left\{s>W_{0}+Z_{1}: \omega_{\mathbf{0}}(s)=0\right\}-\left(W_{0}+Z_{1}\right)
\end{gathered}
$$

e $Y_{1}=Z_{1}+W_{1}$. Note que $Z_{1}$ é uma exponencial com parâmetro $p$ e $W_{1}$ é o tempo de retorno de um passeio aleatório simples em tempo contínuo em $\mathbb{Z}$ com taxas de salto $p$ e 
$q$ (à direita e à esquerda, respectivamente) a partir de 1 à origem e que, sob $\mathbf{P}_{\mathbf{0}}, W_{0}=0$. Para $i \geqslant 1$, suponhamos definidos $Y_{1}, \ldots, Y_{i-1}$ e definamos

$$
\begin{array}{r}
Z_{i}=\inf \left\{s>W_{0}+\sum_{j=1}^{i-1} Y_{j}: \omega_{\mathbf{0}}(s) \neq 0\right\}-\left(W_{0}+\sum_{j=1}^{i-1} Y_{j}\right), \\
W_{i}=\inf \left\{s>W_{0}+\sum_{j=1}^{i-1} Y_{j}+Z_{i}: \omega_{\mathbf{0}}(s)=0\right\}-\left(W_{0}+\sum_{j=1}^{i-1} Y_{j}+Z_{i}\right)
\end{array}
$$

e $Y_{i}=Z_{i}+W_{i}$. Pela propriedade forte de Markov, segue que $Z_{i}$ e $W_{i}$ têm a mesma distribuição de $Z_{1}$ e $W_{1}$, respectivamente. Também pela propriedade forte de Markov, temos que todas as variáveis definidas acima são independentes. Assim, por indução, temos $\left(Y_{i}\right)_{i \geqslant 1}$, uma família de variáveis aleatórias i.i.d. Façamos $T_{0}=W_{0}$ e, para $n \geqslant 1$, $T_{n}=T_{n-1}+Y_{n}$. Além disso, para $t \in \mathbb{R}_{+}$, definamos $C_{t}=\sum_{n=1}^{\infty} \mathbb{1}\left\{T_{n} \leqslant t\right\}$. Note que para $k \in \mathbb{N}$ e $a \geqslant 0$,

$$
\begin{aligned}
\mathbf{P}_{\mathbf{k}}\left(\int_{0}^{t} \mathbb{1}\left\{\omega_{\mathbf{0}}(s)=0\right\} d s<\epsilon t\right)= & \mathbf{P}_{\mathbf{k}}\left(\int_{0}^{t} \mathbb{1}\left\{\omega_{\mathbf{0}}(s)=0\right\} d s<\epsilon t, \mathbf{C}_{t}<\lfloor a t\rfloor\right)+ \\
& +\mathbf{P}_{\mathbf{k}}\left(\int_{0}^{t} \mathbb{1}\left\{\omega_{\mathbf{0}}(s)=0\right\} d s<\epsilon t, \mathbf{C}_{t} \geqslant\lfloor a t\rfloor\right) \\
\leqslant & \mathbf{P}_{\mathbf{k}}\left(\mathbf{C}_{t}<\lfloor a t\rfloor\right)+\mathbf{P}\left(\sum_{j=1}^{\lfloor a t\rfloor} Z_{j}<\epsilon t\right)
\end{aligned}
$$

e que, para $\alpha \in(0,1)$,

$$
\begin{aligned}
\mathbf{P}_{\mathbf{k}}\left(C_{t}<\lfloor a t\rfloor\right) & =\mathbf{P}_{\mathbf{k}}\left(C_{t}<\lfloor a t\rfloor, T_{0}<\alpha t\right)+\mathbf{P}_{\mathbf{k}}\left(C_{t}<\lfloor a t\rfloor, T_{0} \geqslant \alpha t\right) \\
& \leqslant \mathbf{P}_{\mathbf{0}}\left(C_{(1-\alpha) t}<\lfloor a t\rfloor\right)+\mathbf{P}_{\mathbf{k}}\left(T_{0} \geqslant \alpha t\right) \\
& =\mathbf{P}_{\mathbf{0}}\left(T_{\lfloor a t\rfloor}>(1-\alpha) t\right)+\mathbf{P}_{\mathbf{k}}\left(T_{0} \geqslant \alpha t\right)
\end{aligned}
$$

Mas, por uma aplicação da desigualdade de Markov, segue que

$$
\begin{gathered}
\mathbf{P}\left(\sum_{j=1}^{\lfloor a t\rfloor} Z_{j}<\epsilon t\right) \leqslant e^{r \epsilon t}\left(\frac{p}{p+r}\right)^{\lfloor a t\rfloor}, r>-p ; \\
\mathbf{P}\left(T_{\lfloor a t\rfloor}>(1-\alpha) t\right) \leqslant e^{-r(1-\alpha) t}\left[\left(\frac{1-r}{p-r}\right)\left(\frac{1}{2}-\frac{1}{2} \sqrt{1-\frac{4 p q}{(1-r)^{2}}}\right)\right]^{\lfloor a t\rfloor},
\end{gathered}
$$

para $0<r<\min \{p, 1-2 \sqrt{p q}\}$ e, para $k=0, \ldots,\lfloor\delta t\rfloor$, que

$$
\mathbf{P}_{\mathbf{k}}\left(T_{0} \geqslant \alpha t\right) \leqslant e^{-r \alpha t}\left(\frac{1-r-\sqrt{(1-r)^{2}-4 p q}}{2 p}\right)^{\lfloor\delta t\rfloor}, 0<r<1-2 \sqrt{p q}
$$

Tomando valores adequados para $\epsilon, \delta, a, \alpha, r(r \in(0, \min \{p, 1-2 \sqrt{p q}\}), \alpha \in(0,1), \delta=$ 
$\frac{\alpha r p}{q-p}, a=\epsilon=(p-r) r^{2}(1-\alpha)$, por exemplo $)$, segue a existência de $0<\beta<+\infty$ tal que

$$
\sum_{k=1}^{\lfloor\delta t\rfloor} \mathbf{P}_{\mathbf{k}}\left(\int_{0}^{t} \mathbb{1}\left\{\omega_{\mathbf{0}}(s)=0\right\} d s<\epsilon t\right) d t \leqslant \delta t e^{-\beta t}
$$

e assim

$$
\int_{0}^{+\infty} \sum_{k=1}^{\lfloor\delta t\rfloor} \mathbf{P}_{\mathbf{k}}\left(\int_{0}^{t} \mathbb{1}\left\{\omega_{\mathbf{0}}(s)=0\right\} d s<\epsilon t\right) d t<+\infty
$$

o que, por (3.75), conclui a demonstração.

Teorema 3.1. Existe $\mu \in \mathbb{R}_{+}$tal que

$$
\frac{X(t)}{t} \rightarrow \frac{\mathbf{E}\left(\xi_{1}\right)}{\mu} \quad \mathbf{P}_{\mathbf{0}}-\text { q.c. quando } t \nearrow+\infty
$$

Demonstração. Para cada $t \in \mathbb{R}_{+}$, seja $\mathrm{N}_{t}=\inf \left\{n \geqslant 0: \tau_{n}<t\right\}$. É claro que

$$
\tau_{\mathrm{N}_{t}}<t<\tau_{\mathrm{N}_{t}+1}
$$

donde segue, pela Proposição 3.1 e pelo fato de que $\mathrm{N}_{t} \rightarrow \infty \mathbf{P}_{\mathbf{0}}-$ q.c. quando $t \rightarrow \infty$, que

$$
\frac{\mathrm{N}_{t}}{t} \rightarrow \frac{1}{\mu}, \mathbf{P}_{\mathbf{0}}-\text { q.c., quando } t \rightarrow \infty
$$

Daí,

$$
\frac{X(t)}{t}=\frac{\mathrm{x}_{\mathrm{N}_{t}}}{t}=\frac{\mathrm{x}_{\mathrm{N}_{t}}}{\mathrm{~N}_{t}} \times \frac{\mathrm{N}_{t}}{t} \rightarrow \frac{\mathbf{E}\left(\xi_{1}\right)}{\mu}, \mathbf{P}_{\mathbf{0}}-q . c . \text {, quando } t \rightarrow+\infty
$$

\subsection{Teorema central do limite}

A partir dos resultados da seção anterior, encerramos o Capítulo com um Teorema Central do Limite para a posição da partícula.

Teorema 3.2. Sob $\mathbf{P}_{\mathbf{0}}$ e $\mathbf{E}_{\mathbf{0}}\left(\xi_{1}\right)=\mathbf{0}$, existe $0<\mu<\infty$ tal que

$$
\frac{X(t)}{\sqrt{t / \mu}} \Rightarrow N_{d}(\mathbf{0}, \Sigma)
$$

com $\Sigma$ a matriz de covariância de $\xi_{1}$.

Demonstração. Primeiro, ressaltamos que, para $t \in \mathbb{R}_{+}$suficientemente grande,

$$
\frac{X(t)}{\sqrt{t}}=\frac{\mathrm{x}_{t}}{\sqrt{\mathrm{N}_{t}}} \times \sqrt{\frac{\mathrm{N}_{t}}{t}}
$$


e que $\mathrm{N}_{t} / t \rightarrow 1 / \mu=: \gamma, \mathbf{P}_{\mathbf{0}}-q . c .$, com $\mu$ a mesma constante cuja existência foi enunciada na Proposição 3.1. Agora, seja $\epsilon>0$ dado, $u:=\gamma-\epsilon^{2} / 2$ e $v:=\gamma+\epsilon^{2} / 2$. Vale que

$$
\frac{\mathrm{x}_{\mathrm{N}_{t}}}{\sqrt{\mathrm{N}_{t}}}=\frac{\mathrm{x}_{\mathrm{N}_{t}}-\mathrm{x}_{\lfloor u t\rfloor}}{\sqrt{\mathrm{N}_{t}}}+\frac{\mathrm{x}_{\lfloor u t\rfloor}}{\sqrt{\mathrm{N}_{t}}}
$$

Pelo Teorema Central do Limite usual, temos sob $\mathbf{P}$ que

$$
\frac{\mathrm{x}_{\lfloor u t\rfloor}}{\sqrt{\lfloor u t\rfloor}} \Rightarrow N_{d}(\mathbf{0}, \Sigma)
$$

com $\Sigma$ a matriz de covariância de $\xi_{1}$. Além disso, como $\sqrt{\mathrm{N}(t) / a t} \rightarrow \sqrt{\gamma / u}:=\theta_{\epsilon}, \mathbf{P}_{\mathbf{0}}-$ q.c., segue pelo Teorema de Slutsky, que sob $\mathbf{P}_{\mathbf{0}}$,

$$
\frac{\mathrm{x}_{\lfloor u t\rfloor}}{\sqrt{\theta_{\epsilon} \mathrm{N}_{t}}}=\frac{\mathrm{x}_{\lfloor u t\rfloor}}{\sqrt{\lfloor u t\rfloor}} \times \sqrt{\frac{\mathrm{N}_{t}}{\theta_{\epsilon} u t} \times \frac{u t}{\lfloor u t\rfloor}} \Rightarrow N_{d}(\mathbf{0}, \Sigma) .
$$

Agora, vamos mostrar que a primeira parcela do lado direito da Equação (3.93) converge, sob $\mathbf{P}_{\mathbf{0}}$, a zero em probabilidade. Escrevamos $\mathrm{x}_{k}=\left(\mathrm{x}_{k, 1}, \ldots, \mathrm{x}_{k, d}\right), k \in \mathbb{N}$. Temos que

$$
\begin{aligned}
\mathbf{P}_{\mathbf{0}}\left(\left\|\frac{\mathrm{x}_{\mathrm{N}}-\mathrm{x}_{\lfloor u t]}}{\sqrt{\mathrm{N}_{t}}}\right\|>\sqrt{\epsilon}\right)= & \mathbf{P}_{\mathbf{0}}\left(\| \frac{\mathrm{x}_{\mathrm{N}_{t}}-\mathrm{x}_{\lfloor u t]}}{\sqrt{\mathrm{N}_{t}}}|>\sqrt{\epsilon},| \frac{\mathrm{N}_{t}}{t}-\gamma \mid<\frac{\epsilon^{2}}{2}\right) \\
& +\mathbf{P}_{\mathbf{0}}\left(\left\|\frac{\mathrm{x}_{\mathrm{N}}-\mathrm{x}_{\lfloor u t]}}{\sqrt{\mathrm{N}_{t}}}\right\|>\sqrt{\epsilon},\left|\frac{\mathrm{N}_{t}}{t}-\gamma\right| \geqslant \frac{\epsilon^{2}}{2}\right)
\end{aligned}
$$

$\mathrm{e}$

$$
\begin{aligned}
\mathbf{P}_{\mathbf{0}}\left(\left\|\frac{\mathrm{x}_{\mathrm{N}_{t}}-\mathrm{x}_{\lfloor u t]}}{\sqrt{\mathrm{N}_{t}}}\right\|>\sqrt{\epsilon},\left|\frac{\mathrm{N}_{t}}{t}-\gamma\right|<\frac{\epsilon^{2}}{2}\right) & \leqslant \mathbf{P}_{\mathbf{0}}\left(\sup _{u t<\mathrm{N}_{t}<v t}\left\|\frac{\mathrm{x}_{\mathrm{N}_{t}}-\mathrm{x}_{\lfloor u t]}}{\sqrt{\mathrm{N}_{t}}}\right\|>\sqrt{\epsilon}\right) \\
& \leqslant \mathbf{P}_{\mathbf{0}}\left(\sup _{u t<k<v t}\left\|\mathrm{x}_{k}-\mathrm{x}_{\lfloor u t]}\right\|>\sqrt{\epsilon u t}\right) \\
& \leqslant \mathbf{P}_{\mathbf{0}}\left(\bigcup_{i=1}^{d}\left\{\sup _{u t<k<v t}\left|\mathrm{x}_{k, i}-\mathrm{x}_{\lfloor u t], i}\right|>\sqrt{\epsilon u t}\right\}\right) \\
& \leqslant \sum_{i=1}^{d} \mathbf{P}_{\mathbf{0}}\left(\sup _{u t<k<v t}\left|\mathrm{x}_{k, i}-\mathrm{x}_{\lfloor u t], i}\right|>\sqrt{\epsilon u t}\right) \\
& \leqslant \frac{\epsilon \operatorname{Tr}(\Sigma)}{\gamma-\epsilon^{2} / 2},
\end{aligned}
$$

em que, dada $A$ uma matriz de orderm $d, \operatorname{Tr}(A)$ indica o traço de $A$. Na última passagem de (3.97), usamos a Desiguldade Maximal de Kolmogorov (vide [Durret, 2010], Capítulo 2, Seção 2.5, Teorema 2.5.2, pg. 79). Assim, como $\mathrm{N}_{t} / t \rightarrow 1 / \mu=: \gamma, \mathbf{P}_{\mathbf{0}}-$ q.c.,

$$
\lim _{t \rightarrow+\infty} \mathbf{P}_{\mathbf{0}}\left(\left\|\frac{\mathrm{x}_{\mathrm{N}_{t}}-\mathrm{x}_{\lfloor u t]}}{\sqrt{\mathrm{N}_{t}}}\right\|>\sqrt{\epsilon}\right) \leqslant \frac{\epsilon \operatorname{Tr}(\Sigma)}{\gamma-\epsilon^{2} / 2},
$$

que tende a zero quando $\epsilon \searrow 0$. Assim, tomando limites em $t \nearrow+\infty$ e em $\epsilon \searrow 0$, obtemos sob $\mathbf{P}_{\mathbf{0}}$ pelo Teorema de Slutsky, que

$$
\frac{X(t)}{\sqrt{t}} \Rightarrow \sqrt{\gamma} N_{d}(\mathbf{0}, \Sigma)
$$


como queríamos demonstrar.

Na demonstração acima, usamos a Desigualdade Maximal de Kolmogorov em uma das passagens em (3.97); que por sua vez exige a média nula para o passeio. Pretendemos abordar o caso de média não-nula em trabalhos futuros via argumentos de regeneração. 


\section{Capítulo 4}

\section{Extensões}

No Capítulo anterior, apresentamos uma Lei Forte dos Grandes Números e um Teorema Central do Limite sob $\mathbf{P}_{\mathbf{0}}$. Nesse capítulo, apresentamos uma extensão destes resultados sob $\mathbf{P}_{\hat{\mu}_{0}}, \hat{\mu}_{0} \leq \hat{\nu}$. Também discorremos sobre o caso em que $\left(\omega_{\mathbf{x}}\right)_{\mathbf{x} \in \mathbb{Z}^{d}}$ são não ergódicos e provamos a localização do passeio sob o caso $\left(\omega_{\mathbf{x}}\right)_{\mathbf{x} \in \mathbb{Z}^{d}}$ transientes.

\subsection{Medida inicial}

Proposição 4.1. Suponha $d \leqslant 2, \hat{\mu}_{0} \leq \hat{\nu}$ e $\xi_{1}$ limitada e simétrica. Então, quando $t \nearrow+\infty$,

$$
\mathbf{P}_{\hat{\mu}_{0}}\left(\left\|\frac{X(t)}{t}\right\|>\epsilon\right) \rightarrow 0, \text { para todo } \epsilon>0
$$

e, sob $\mathbf{P}_{\hat{\mu}_{0}}$, existe $\mu \in \mathbb{R}_{+}$tal que

$$
\frac{X(t)}{\sqrt{t / \mu}} \Rightarrow N_{d}(\mathbf{0}, \Sigma),
$$

com $\Sigma$ a matriz de covariância de $\xi_{1}$. Se $d=1$, os resultados permanecem sob $\xi_{1}$ assimétrica.

Demonstração. Vamos considerar um acoplamento de duas versões do processo, a primeira sob $\mathbf{P}_{\mathbf{0}}$ e a segunda sob $\mathbf{P}_{\hat{\nu}}$. Ambas as versões têm a distribuição dos respectivos saltos no espaço $\mathbb{Z}^{d}$ dadas por $\pi$; que por sua vez tem suporte $\mathbb{Z}^{d} \backslash\{\mathbf{0}\}$ e é tal que $\pi(\mathbf{k})=\pi(-\mathbf{k})$ e $\pi(\mathbf{k})=0$ quando $|\mathbf{k}| \geqslant B$ (para $d=2,|\cdot|$ indica a distância de Mahalanobis), $\mathbf{k} \in \mathbb{Z}^{d}, B \in \mathbb{R}_{+}, d \leqslant 2$. Vamos mostrar que, sob tal acoplamento, as partículas eventualmente coalescem, o que será suficiente para garantir os resultados:

Sejam $\left(X^{0}(t), \omega^{0}(t)\right)_{t \in \mathbb{R}_{+}}$e $\left(X^{1}(t), \omega^{1}(t)\right)_{t \in \mathbb{R}_{+}}$duas versões independentes do processo, a primeira sob $\mathbf{P}_{\mathbf{0}}$ e a segunda sob $\mathbf{P}_{\hat{\mu}_{0}}, \hat{\mu}_{0} \leq \hat{\nu}$. De modo respectivo, sejam $\left(\xi_{n}^{i}\right)_{n \in \mathbb{N}_{*}}$ e $\left(\mathcal{N}_{\mathbf{x}}^{i}\right)_{\mathbf{x} \in \mathbb{Z}^{d}}$ os elementos aleatórios usados, de acordo com a primeira construção do modelo, para a obtenção de $\left(X^{i}(t)\right)_{t \in \mathbb{R}_{+}}$, $i=0,1$. $\left(\xi_{n}^{0}\right)_{n \in \mathbb{N}_{*}}$ e $\left(\xi_{n}^{1}\right)_{n \in \mathbb{N}_{*}}$ são independentes entre si e todas com distribuição $\pi$, em que $\pi$ tem suporte $\mathbb{Z}^{d} \backslash\{\mathbf{0}\}$ com $\pi(\mathbf{k})=\pi(-\mathbf{k})$ e $\pi(\mathbf{k})=0$ quando $|\mathbf{k}| \geqslant B, \mathbf{k} \in \mathbb{Z}^{d}, B \in \mathbb{R}_{+}, d \leqslant 2$. Denotemos também por $\mathrm{N}_{s}^{i}$ o número de saltos de $\left(X^{i}(t)\right)_{t \in \mathbb{R}_{+}}$até o tempo $s \in \mathbb{R}_{+}, i=0,1$. Definamos ainda, 
para cada $\mathbf{x} \in \mathbb{Z}^{d}$

$$
\mathrm{T}_{\mathbf{x}}=\inf \left\{s>0: \omega_{\mathbf{x}}^{1}(s)=\omega_{\mathbf{x}}^{0}(s)\right\} .
$$

Agora, consideremos $\left(\xi_{n}\right)_{n \in \mathbb{N}_{*}}$ i.i.d. com distribuição $\pi$ independentes dos objetos descritos acima e seja $(X(t))_{t \in \mathbb{R}_{+}}$um passeio aleatório a tempo contínuo em $\mathbb{Z}^{d}$ auxiliar definido do seguinte modo:

Façamos $\tau_{0}=0$ e $X(0)=X^{1}(0)=0$. Definamos

$$
\mathbb{I}_{0}=1-\mathbb{1}\left\{\mathrm{T}_{X(0)} \leqslant 0, X(0)=X^{0}(0)\right\}
$$

e façamos

$$
\tau_{1}=\inf \left\{s>0: \mathcal{N}_{X(0)}^{\mathbb{I}_{0}} \cap\left(Q_{X(0)} \times(0, s]\right) \neq \varnothing\right\} .
$$

Para $t \in\left(\tau_{0}, \tau_{1}\right), X(t)=X(0) \mathrm{e}$

$$
X\left(\tau_{1}\right)=X(0)+\mathbb{I}_{0} \xi_{1}+\left(1-\mathbb{I}_{0}\right) \xi_{\mathrm{N}^{0}\left(\tau_{1}^{-}\right)+1}^{0},
$$

com $\mathrm{N}^{0}\left(t^{-}\right):=\lim _{s \nearrow t} \mathrm{~N}_{s}^{0}$. Para $n \geqslant 2$, suponhamos definidos $\tau_{1}, \ldots, \tau_{n-1}$ e $X(t)$, para $t \leqslant \tau_{n-1}$. Definamos

$$
\mathbb{I}_{n-1}=1-\mathbb{1}\left\{\mathrm{T}_{X\left(\tau_{n-1}\right)} \leqslant \tau_{n-1}, X\left(\tau_{n-1}\right)=X^{0}\left(\tau_{n-1}\right)\right\}
$$

e façamos

$$
\tau_{n}=\inf \left\{s>\tau_{n-1}: \mathcal{N}_{X\left(\tau_{n-1}\right)}^{\mathbb{I}_{n-1}} \cap\left(Q_{X\left(\tau_{n-1}\right)} \times\left(\tau_{n-1}, s\right]\right) \neq \varnothing\right\} .
$$

Para $t \in\left(\tau_{n-1}, \tau_{n}\right), X(t)=X\left(\tau_{n-1}\right)$ e

$$
X\left(\tau_{n}\right)=X\left(\tau_{n-1}\right)+\mathbb{I}_{n-1} \xi_{n}+\left(1-\mathbb{I}_{n-1}\right) \xi_{\mathrm{N}^{0}\left(\tau_{n}^{-}\right)+1}^{0}
$$

Por indução, fica definido $(X(t))_{t \in \mathbb{R}_{+}}$. Da construção, não é difícil ver que $\left(X^{0}(t), X(t)\right)_{t \in \mathbb{R}_{+}}$é um acoplamento de $\left(X^{0}(t)\right)_{t \in \mathbb{R}_{+}}$e $\left(X^{1}(t)\right)_{t \in \mathbb{R}_{+}}$. Subjacente à $(X(t))_{t \in \mathbb{R}_{+}}$, temos o meio $\omega$ definido pela seguinte regra:

$$
\omega_{\mathbf{x}}(s)= \begin{cases}\omega_{\mathbf{x}}^{1}(s), & s<\mathrm{T}_{\mathbf{x}} \\ \omega_{\mathbf{x}}^{0}(s), & s \geqslant \mathrm{~T}_{\mathbf{x}},\end{cases}
$$

i.e., $\left(\omega_{\mathbf{x}}^{0}, \omega_{\mathbf{x}}\right)$ é um acoplamento clássico de $\omega_{\mathbf{x}}^{0}$ e $\omega_{\mathbf{x}}^{1}, \mathbf{x} \in \mathbb{Z}^{d}$. Assim, sob tal meio, $(X(t))_{t \in \mathbb{R}_{+}}$é um passeio aleatório com tempos de saltos $\left(\tau_{n}\right)_{n \in \mathbb{N}}$ que evolui de modo independente de $\left(X^{0}(t)\right)_{t \in \mathbb{R}_{+}}$, a menos do caso em que sua trajetória coincida com a de $\left(X^{0}(t)\right)_{t \in \mathbb{R}_{+}}$numa posição $\mathbf{x} \in \mathbb{Z}^{d}$ pós o tempo $T_{\mathbf{x}}$ : nesse cenário, a partir desse encontro, as duas partículas permanecem juntas até que a visita, caso exista, a uma posição com componentes do meio não acopladas as separem. Denotemos por $\mathbb{P}$ a medida de probabilidade subjacente à $\left(X^{0}(t), X(t)\right)_{t \in \mathbb{R}_{+}}$. Note que, para $\epsilon>0$,

$$
\mathbf{P}_{\hat{\mu}_{0}}\left(\left|\frac{X^{1}(t)}{t}\right|>\epsilon\right)=\mathbb{P}\left(\left|\frac{X(t)}{t}\right|>\epsilon, X(t)=X^{0}(t)\right)+\mathbb{P}\left(\left|\frac{X(t)}{t}\right|>\epsilon, X(t) \neq X^{0}(t)\right)
$$

e, para $E$ um Boreliano de $\mathbb{R}^{d}$ qualquer, 


$$
\mathbf{P}_{\hat{\mu}_{0}}\left(\frac{X^{1}(t)}{\sqrt{t / \mu}} \in E\right)=\mathbb{P}\left(\frac{X(t)}{\sqrt{t / \mu}} \in E, X(t)=X^{0}(t)\right)+\mathbb{P}\left(\frac{X(t)}{\sqrt{t / \mu}} \in E, X(t) \neq X^{0}(t)\right) .
$$

Logo, para findar a prova, basta mostrar que $\mathbb{P}\left(X(t)=X^{0}(t)\right)$ tende a um quando $t \nearrow+\infty$, uma vez que em $\left\{X(t)=X^{0}(t)\right\}, X(t)$ sob $\mathbb{P}$ equivale a $X^{0}(t)$, que é sob $\mathbf{P}_{\mathbf{0}}$ e cujo comportamento assintótico já é conhecido.

Vamos então mostrar que $\mathbb{P}\left(X(t)=X^{0}(t)\right) \rightarrow 1$ quanto $t \nearrow+\infty$. Seja $(\mathrm{D}(s))_{s \in \mathbb{R}_{+}}$definido por $\mathrm{D}(s):=X(s)-X^{0}(s), s \in \mathbb{R}_{+}$. Denotemos por $\left(\mathrm{d}_{n}\right)_{n \in \mathbb{N}}$ a cadeia imersa de $(\mathrm{D}(s))_{s \in \mathbb{R}_{+}}$e consideremos o evento

$$
\mathrm{A}_{0}=\lim \sup \left\{\mathrm{d}_{n}=0\right\}, \quad n \in \mathbb{N},
$$

que corresponde a infinitos retornos de $\left(\mathrm{d}_{n}\right)_{n \in \mathbb{N}}$ à origem. Agora, para $U \subset \mathbb{Z}^{d}$, façamos $\omega_{U}:=$ $\left(\omega_{\mathbf{x}}\right)_{\mathbf{x} \in U}$ e, para $k \in \mathbb{N}_{*}, \Lambda_{k}:=\omega_{U_{k}}$ em que $U_{k}:=\{-B k, \ldots, B k\}^{d}$. De modo análogo, para $U \subset \mathbb{Z}^{d}$ e $k \in \mathbb{N}_{*}$, ficam definidos $\omega_{U}^{0}$ e $\Lambda_{k}^{0}$. Definamos

$$
\lambda_{k}:=\inf \left\{s>0: \Lambda_{k}(s)=\Lambda_{k}^{0}(s)\right\}, \quad k \in \mathbb{N},
$$

e, para $m \in \mathbb{N}_{*}$ e $\alpha \in(0,2)$, consideremos também os eventos

$$
\mathrm{A}_{m}=\left\{\lambda_{2 m}<m^{\alpha}\right\} \quad \text { e } \quad \mathrm{B}_{m}=\left\{\max _{0 \leqslant u \leqslant m^{\alpha}}\left\{\left|X^{0}(u)\right|,|X(u)|\right\} \leqslant B m\right\}
$$

que indicam, de modo respectivo, o acoplamento do meio na região $U_{2 m}$ antes do tempo $m^{\alpha}$ e o confinamento das partículas $\left(X^{0}(t)\right)_{t \in \mathbb{R}_{+}}$e $(X(t))_{t \in \mathbb{R}_{+}}$em $U_{m} \times\left[0, m^{\alpha}\right]$. Por fim, consideremos o evento

$$
\mathrm{C}_{m}=\bigcap_{k \in \mathbb{N}_{*}} \mathrm{C}_{m, k}
$$

com os eventos $\left\{\mathrm{C}_{m, k}\right\}_{k \in \mathbb{N}_{*}}$ definidos da seguinte maneira:

Façamos

$$
\kappa_{0}=\inf \left\{s>0:\left\{X(s), X^{0}(s)\right\} \nsubseteq U_{2 m}\right\}
$$

e definamos

$$
\mathrm{C}_{m, 1}:=\left\{\Lambda_{4 m}\left(\kappa_{0}\right)=\Lambda_{4 m}^{0}\left(\kappa_{0}\right)\right\}
$$

Agora, dado $\kappa_{0}$, façamos

$$
\kappa_{1}:=\inf \left\{s>\kappa_{0}:\left\{X(s), X^{0}(s)\right\} \nsubseteq U_{4 m}\right\}
$$

e definamos

$$
\mathrm{C}_{m, 2}:=\left\{\Lambda_{8 m}\left(\kappa_{1}\right)=\Lambda_{8 m}^{0}\left(\kappa_{1}\right)\right\} .
$$


De modo análogo, para $i \geqslant 2$, dado $\kappa_{i-1}$, façamos

$$
\kappa_{i}:=\inf \left\{s>\kappa_{i-1}:\left\{X(s), X^{0}(s)\right\} \nsubseteq U_{m 2^{i+1}}\right\}
$$

e definamos

$$
\mathrm{C}_{m, i+1}:=\left\{\Lambda_{2^{i+2} m}\left(\kappa_{i}\right)=\Lambda_{2^{i+2} m}^{0}\left(\kappa_{i}\right)\right\} .
$$

Em palavras, $C_{m, i+1}$ representa o acoplamento dos ambientes $\omega^{0}$ e $\omega$ nos sítios $U_{m 2^{i+2}}$ antes do tempo $\kappa_{i}$, que por sua vez, representa o tempo necessário para que uma das partículas $\left(X^{0}(s)\right)_{s \in \mathbb{R}_{+}}$ ou $(X(s))_{s \in \mathbb{R}_{+}}$visite um sítio fora da região $U_{m 2^{i+1}}, i \in \mathbb{N}$.

É claro que $\mathrm{E}_{m}:=\mathrm{A}_{0} \cap \mathrm{A}_{m} \cap \mathrm{B}_{m} \cap \mathrm{C}_{m} \subset\left\{\exists t_{0} \in \mathbb{R}_{+}: X(t)=X^{0}(t) \forall t \geqslant t_{0}\right\}$. De fato, em $\mathrm{E}_{m}$, pós o tempo $m^{\alpha}$, as partículas visitam apenas regiões em que os meios $\omega$ e $\omega^{0}$ já estão acoplados. A partir daí, no próximo encontro, que irá ocorrer por $A_{0}$, elas coalescem. Assim,

$$
\lim _{t \nearrow+\infty} \mathbb{P}\left(X(t)=X^{0}(t)\right) \geqslant \mathbb{P}\left(\mathrm{E}_{m}\right), \quad m \in \mathbb{N}_{*} .
$$

Para findar, vamos mostra que a probabilidade de $\mathrm{E}_{m}$ pode ser tomada tão alta quanto se queira, a depender de $m$.

A partir de agora, vamos supor, sem perda de generalidade, que $\left\{\mathbf{x} \in \mathbb{Z}^{d}: \pi(\mathbf{x})>0\right\}$ não seja subconjunto de um hiperplano $d^{*}$-dimensional, $d^{*}<d$, de modo que $\left(\mathrm{x}_{n}\right)_{n \in \mathbb{N}}$ seja genuinamente $d$-dimensional; e que $B=1$. Da simetria de $\xi_{1}$, não é difícil ver que $\left(\mathrm{d}_{n}\right)_{n \in \mathbb{N}}$ é um passeio aleatório em $\mathbb{Z}^{d}$ com média nula. Como, por construção, $\mathbf{E}\left(\left\|\xi_{1}\right\|^{2}\right)<+\infty$, é bem conhecido que $\left(\mathrm{d}_{n}\right)_{n \in \mathbb{N}}$ é recorrente nulo(vide [Spitzer, 1976], Capítulo 2, Seção 8, pg. 83), de modo que

$$
\mathbb{P}\left(\mathrm{A}_{0}\right)=1
$$

e, assim, $\mathbb{P}\left(\mathrm{E}_{m}\right)=\mathbb{P}\left(\mathrm{A}_{m} \cap \mathrm{B}_{m} \cap \mathrm{C}_{m}\right)$. Agora, façamos $\mathrm{H}_{x}=\inf \left\{s>0: \omega_{\mathbf{x}}(s)=0\right\}, \mathbf{x} \in \mathbb{Z}$. É claro que

$$
\lambda_{m} \leqslant \max \left\{\mathrm{H}_{\mathbf{x}}: \mathbf{x} \in U_{2 m}\right\}
$$

Daí,

$$
\mathbb{P}\left(\mathrm{A}_{m}\right) \geqslant\left[1-\mathbb{P}\left(\mathrm{H}_{0}>m^{\alpha}\right)\right]^{(4 m+1)^{d}} .
$$

Fazendo $\mathbb{P}_{k}(\cdot):=\mathbb{P}\left(\cdot \mid \omega_{0}(0)=k\right)$ e $\mathbb{E}_{k}(\cdot):=\mathbb{E}\left(\cdot \mid \omega_{0}(0)=k\right), k \in \mathbb{N}$, temos

$$
\mathbb{P}\left(\mathrm{H}_{\mathbf{0}}>m^{\alpha}\right)=\sum_{k \in \mathbb{N}_{*}}(1-\rho) \rho^{k} \mathbb{P}_{k}\left(\mathrm{H}_{\mathbf{0}}>m^{\alpha}\right)
$$

e para $u \in D:=D\left(\mathrm{H}_{\mathbf{0}}\right)=[0,1-2 \sqrt{p q})$, pela desigualdade de Chernoff e pela Propriedade Forte de Markov,

$$
\mathbb{P}_{k}\left(\mathrm{H}_{\mathbf{0}}>m^{\alpha}\right)=\mathbb{P}_{k}\left(e^{u \mathrm{H}_{0}}>e^{u m^{\alpha}}\right) \leqslant \inf _{u \in D} \mathbb{E}_{1}\left(e^{u \mathrm{H}_{0}}\right)^{k} e^{-u m^{\alpha}},
$$

com

$$
\mathbb{E}_{1}\left(e^{u \mathrm{H}_{0}}\right)=\left(1-u-\sqrt{(1-u)^{2}-4 p q}\right) / 2 p \leqslant \sqrt{\frac{q}{p}}=\rho^{-1 / 2}, \quad u \in D .
$$


Assim, para $u \in D$,

$$
\mathbb{P}\left(\mathbf{H}_{\mathbf{0}}>m^{\alpha}\right) \leqslant(1-\rho) e^{-u m^{\alpha}} \sum_{k \in \mathbb{N}_{*}} \rho^{k / 2}=\sqrt{\rho}(1+\sqrt{\rho}) e^{-u m^{\alpha}}
$$

de onde segue que

$$
\mathbb{P}\left(\mathrm{A}_{m}\right) \geqslant\left(1-\gamma e^{-\beta m^{\alpha}}\right)^{(4 m+1)^{d}}, \quad \gamma:=\sqrt{\rho}(1+\sqrt{\rho}), \beta=1-2 \sqrt{p q} .
$$

É fácil ver que $\mathrm{B}_{m}$ é mais provável sob $d=2$ do que sob $d=1$. Além disso, por construção, independente de $\mathrm{A}_{m}$, o número de saltos de $(X(s))_{s \in \mathbb{R}_{+}}$sob $d=1$ é dominado pelo número de saltos de um passeio aleatório a tempo contínuo usual com incrementos distribuídos de acordo com $\pi$. O mesmo vale para $\left(X^{0}(s)\right)_{s \in \mathbb{R}_{+}}$. Assim, seja agora $(\mathrm{Y}(s))_{s \in \mathbb{R}_{+}}$um passeio aleatório a tempo contínuo usual com incrementos no espaço $\mathbb{Z}$ distribuídos de acordo com $\pi$ e denotemos por $\left(\mathrm{M}_{s}\right)_{s \in \mathbb{R}_{+}}$e $\left(\mathrm{y}_{k}\right)_{k \in \mathbb{N}}$ o $P P P(1)$ que determina seus instantes de saltos e sua cadeia imersa, respectivamente. Temos então que

$$
\mathbb{P}\left(\mathrm{B}_{m} \mid \mathrm{A}_{m}\right) \geqslant \mathbb{P}\left(\max _{0 \leqslant u \leqslant m^{\alpha}}|\mathrm{Y}(u)| \leqslant m\right)^{2} .
$$

Mas

$$
\begin{aligned}
\mathbb{P}\left(\max _{0 \leqslant u \leqslant m^{\alpha}}|\mathrm{Y}(u)| \leqslant m\right) & =\mathbb{P}\left(\mathrm{M}_{m^{\alpha}} \leqslant m\right)+\sum_{k>m} \mathbb{P}\left(\max _{0 \leqslant u \leqslant m^{\alpha}}|\mathrm{Y}(u)| \leqslant n \mid \mathrm{M}_{m^{\alpha}}=k\right) \mathbb{P}\left(\mathrm{M}_{m^{\alpha}}=k\right) \\
& =\mathbb{P}\left(\mathrm{M}_{m^{\alpha}} \leqslant m\right)+\sum_{k>m} \mathbb{P}\left(\max _{0 \leqslant l \leqslant k}\left|\mathrm{y}_{l}\right| \leqslant m\right) \mathbb{P}\left(\mathrm{M}_{m^{\alpha}}=k\right) \\
& =1-\sum_{k>m} \mathbb{P}\left(\max _{0 \leqslant l \leqslant k}\left|\mathrm{y}_{l}\right| \geqslant m+1\right) \mathbb{P}\left(\mathrm{M}_{m^{\alpha}}=k\right)
\end{aligned}
$$

Pela Desigualdade Maximal de Kolmogorov,

$$
\mathbb{P}\left(\max _{0 \leqslant l \leqslant k}\left|\mathrm{y}_{l}\right| \geqslant m+1\right) \leqslant \frac{\operatorname{Var}\left(\mathrm{y}_{k}\right)}{(m+1)^{2}}=\frac{k \mathbf{E}\left(\xi_{1}^{2}\right)}{(m+1)^{2}}
$$

donde segue que

$$
\mathbb{P}\left(\max _{0 \leqslant u \leqslant m^{\alpha}}|\mathrm{Y}(u)| \leqslant m\right) \geqslant 1-\frac{\mathbf{E}\left(\xi_{1}^{2}\right)}{(m+1)^{2}} \sum_{k>m} k \mathbb{P}\left(\mathrm{M}_{m^{\alpha}}=k\right) \geqslant 1-\frac{m^{\alpha} \mathbf{E}\left(\xi_{1}^{2}\right)}{(m+1)^{2}},
$$

e concluímos que

$$
\mathbb{P}\left(\mathrm{B}_{m} \mid \mathrm{A}_{m}\right) \geqslant\left(1-\frac{m^{\alpha} \mathbf{E}\left(\xi_{1}^{2}\right)}{(m+1)^{2}}\right)^{2}
$$

Vamos agora ao cálculo de $\mathbb{P}\left(\mathrm{C}_{m} \mid \mathrm{A}_{m} \cap \mathrm{B}_{m}\right)$. Em $A_{m} \cap B_{m}$, as duas partículas permanecem na região $U_{m}$ e há acoplamento dos meios nos $(4 m+1)^{d}$ sítios de $U_{2 m}$ antes do tempo $m^{\alpha}$. Desse modo, 


$$
\begin{aligned}
\mathbb{P}\left(\mathrm{C}_{m, 1}^{c} \mid \mathrm{A}_{m} \cap \mathrm{B}_{m}\right) & =\int_{m^{\alpha}}^{\infty}\left[1-\mathbb{P}\left(\mathrm{C}_{m, 1} \mid \mathrm{A}_{m} \cap \mathrm{B}_{m}, \kappa_{0}=s\right)\right] \mathbb{P}\left(\kappa_{0} \in \mathrm{d} s\right) \mathrm{d} s \\
& \leqslant \int_{m^{\alpha}}^{\infty}\left[1-\left(1-\gamma e^{-\beta s}\right)^{(8 m+1)^{d}-(4 m+1)^{d}}\right] \mathbb{P}\left(\kappa_{0} \in \mathrm{d} s\right) \mathrm{d} s \\
& \leqslant \int_{0}^{\infty}\left[1-\left(1-\gamma e^{-\beta s}\right)^{8^{d} m^{d}}\right] \mathbb{P}\left(\kappa_{0} \in \mathrm{d} s\right) \mathrm{d} s \\
& =\mathbb{E}\left(1-\left(1-\gamma e^{-\beta \kappa_{0}}\right)^{8^{d} m^{d}}\right)
\end{aligned}
$$

com as duas desigualdades decorrentes, respectivamente, de argumentos análogos aos usados para a obtenção de $(4.31)$ do fato de $(8 m+1)^{d}-(4 m+1)^{d} \leqslant 8^{d} m^{d}$ para $m \in \mathbb{N}$ e $d \leqslant 2$. Como $1-x \geqslant e^{-2 x}$ vale para $0<x<0,5$ e $1-e^{-x} \leqslant x$, temos que

$$
\begin{aligned}
\mathbb{E}\left(1-\left(1-\frac{\gamma}{e^{\beta \kappa_{0}}}\right)^{8^{d} m^{d}}\right) & =\mathbb{E}\left(1-\left(1-\frac{\gamma}{e^{\beta \kappa_{0}}}\right)^{8^{d} m^{d}} ; e^{\beta \kappa_{0}}>2 \gamma\right) \\
& +\mathbb{E}\left(1-\left(1-\frac{\gamma}{e^{\beta \kappa_{0}}}\right)^{8^{d} m^{d}} ; e^{\beta \mathbf{k}_{0}}<2 \gamma\right) \\
& \leqslant \mathbb{E}\left(1-e^{-2 \gamma 8^{d} m^{d} / e^{\beta \kappa_{0}}} ; e^{\beta \kappa_{0}}>2 \gamma\right)+\mathbb{P}\left(e^{\beta \kappa_{0}}<2 \gamma\right) \\
& \leqslant \mathbb{E}\left(\frac{2^{3 d+1} \gamma m^{d}}{e^{\beta \kappa_{0}}}\right)+\mathbb{P}\left(e^{-\beta \kappa_{0}}>\frac{1}{2 \gamma}\right) .
\end{aligned}
$$

Mas $\kappa_{0} \geqslant \sum_{k=1}^{2 m} \mathrm{~V}_{0, k} \geqslant \sum_{k=1}^{m} \mathrm{~V}_{0, k}$, com $\left\{\mathrm{V}_{0, k}\right\}_{k \in \mathbb{N}_{*}}$ exponenciais padrões independentes entre si e de todo o resto (é necessário ao menos $2 m$ saltos para que uma das partículas deixe a região $U_{2 m}$ ). Assim, pela desigualdade de Chernoff, segue que

$$
\begin{aligned}
\mathbb{P}\left(\mathrm{C}_{m, 1}^{c} \mid \mathrm{A}_{m} \cap \mathrm{B}_{m}\right) & \leqslant \mathbb{E}\left(\frac{2^{3 d+1} m^{d} \gamma}{\beta \sum_{k=1}^{m} \mathrm{~V}_{0, i}}\right)+\mathbb{P}\left(e^{-\beta \sum_{k=1}^{m} \mathrm{~V}_{0, i}}>\frac{1}{2 \gamma}\right) \\
& \leqslant \frac{\left(2^{3 d+1} m^{d}+2\right) \gamma}{(1+\beta)^{m}} \leqslant \frac{2^{3 d+2} m^{d} \gamma}{(1+\beta)^{m}} .
\end{aligned}
$$

A partir dos mesmos argumentos dados em (4.37) (dessa vez condicionando em $\kappa_{i-1}$ ) e do fato de que $\kappa_{i-1} \geqslant \sum_{k=1}^{m 2^{i}} \mathrm{~V}_{i, k}$, com $\left\{\mathrm{V}_{i, k}\right\}_{k \in \mathbb{N}_{*}}$ exponenciais padrões independentes entre si e de todo o resto, segue para $i \geqslant 2$ que

$$
\begin{aligned}
\mathbb{P}\left(\mathrm{C}_{m, i}^{c} \mid \mathrm{A}_{m} \cap \mathrm{B}_{m}\right) & \leqslant \mathbb{E}\left(\frac{2^{i+2} m^{d} \gamma}{e^{\beta \kappa_{i-1}}}\right)+\mathbb{P}\left(e^{-\beta \kappa_{i-1}}>\frac{1}{2 \gamma}\right) \\
& \leqslant \frac{\left(2^{(2+i) d+1} m^{d}+2\right) \gamma}{(1+\beta)^{2^{i} m}} \\
& \leqslant \frac{2^{(2+i) d+2} m^{d} \gamma}{(1+\beta)^{i m}} .
\end{aligned}
$$


Decorrente disso, segue que

$$
\begin{aligned}
\mathbb{P}\left(\mathrm{C}_{m} \mid \mathrm{A}_{m} \cap \mathrm{B}_{m}\right) & \geqslant 1-\sum_{i \geqslant 1} \mathbb{P}\left(\mathrm{C}_{m, i}^{c} \mid \mathrm{A}_{m} \cap \mathrm{B}_{m}\right) \\
& \geqslant 1-2^{2 d+2} m^{d} \gamma \sum_{i \geqslant 1}\left(\frac{2^{d}}{(1+\beta)^{m}}\right)^{i} \geqslant 1-\frac{2^{3 d+3} m^{d} \gamma}{(1+\beta)^{m}},
\end{aligned}
$$

para todo $m>(d+1) \ln (2) /(\ln (1+\beta))$. Das expressões acima, segue que

$$
\lim _{m \rightarrow \infty} \mathbb{P}\left(\mathrm{E}_{m}\right)=\lim _{m \rightarrow \infty} \mathbb{P}\left(\mathrm{A}_{m}\right) \mathbb{P}\left(\mathrm{B}_{m} \mid \mathrm{A}_{m}\right) \mathbb{P}\left(\mathrm{C}_{m} \mid \mathrm{A}_{m} \cap \mathrm{B}_{m}\right)=1
$$

o que é suficiente para garantir que $X$ e $X^{0}$ coalescem.

Agora, se $d=1$, mesmo sob $\xi_{1}$ assimétrica, o resultado acima permanece válido. De fato, sob $\xi_{1}$ assimétrica, a única mudança em relação aos argumentos anteriores se dá no comportamento de $\left(\mathrm{d}_{n}\right)_{n \in \mathbb{N}} ;$ que agora é um passeio aleatório em $\mathbb{Z}$ não homogêneo no tempo, cujo incremento no tempo $n$ tem distribuição igual a de $\xi_{1}$ ou igual a de $-\xi_{1}$. Como $\mathbf{E}\left(\xi_{1}^{2}\right)<+\infty$, pode-se mostrar, para $d=1$, que $\left(\mathrm{d}_{n}\right)_{n \in \mathbb{N}}$ retornará infinitas vezes à origem (veja [Durrett et al., 1991], Teorema 1), i.e., ainda vale $\mathbb{P}\left(\mathrm{A}_{0}\right)=1$. Os demais eventos independem de $\xi_{1}$ ser simétrica.

Por fim, ressaltamos que a extensão para $\hat{\mu}_{0} \leq \hat{\nu}$ segue sem maiores dificuldades do fato de que $\mathrm{H}_{x}$ sob $\hat{\mu}_{0}$ é dominada estocasticamente por $\mathrm{H}_{x}$ sob $\hat{\nu}$; o que pode ser verificado via acoplamento clássico de processos de nascimento e morte.

Para o caso em que $\mathbf{E}\left\|\xi_{1}\right\|^{2}$, mas ilimitada, o mesmo argumento dado acima deve funcionar. Para tal, será necessário utilizar cotas mais refinadas para os tempos esperados para a fuga das regiões meio-acopladas usadas em 4.38 e (4.39). Pretendemos apresentar tal extensão em trabalhos futuros.

Proposição 4.2. Suponha $\xi_{1}$ limitada $e \hat{\mu}_{0} \leq \hat{\nu}$. Então, para $d \geqslant 3$, quando $t \nearrow+\infty$,

$$
\mathbf{P}_{\hat{\mu}_{0}}\left(\left\|\frac{X(t)}{t}\right\|>\epsilon\right) \rightarrow 0, \text { para todo } \epsilon>0
$$

e, sob $\mathbf{P}_{\hat{\mu}_{0}}$, existe $\mu \in \mathbb{R}_{+}$tal que

$$
\frac{X(t)}{\sqrt{t / \mu}} \Rightarrow N_{d}(\mathbf{0}, \Sigma)
$$

com $\Sigma$ a matriz de covariância de $\xi_{1}$.

Demonstração. Para a prova, fixado $\epsilon>0$ arbitrariamente, construiremos conjuntamente duas versões do processo, digamos $\left(\omega^{0}, X^{0}\right)$ e $(\omega, X)$; a primeira sob $\mathbf{P}_{\mathbf{0}}$, a segunda sob $\mathbf{P}_{\hat{\nu}}$ e de tal modo que, com probabilidade $1-\epsilon,\left\|X(t)-X^{0}(t)\right\|<+\infty$ para todo $t \in \mathbb{R}_{+}$, donde seguirá o resultado.

Inicialmente, sejam $\omega^{0}$ e $\omega^{1}$ duas versões independentes do meio, a primeira com $\omega^{0}(0)=\mathbf{0}$ e a segunda com medida inicial $\hat{\nu}$. Façamos ainda uma terceira versão $\tilde{\omega}$ tal $\left(\omega^{0}, \tilde{\omega}\right)$ é um acoplamento clássico de $\omega^{0}$ e $\omega^{1}$ no sentido de que $\left(\omega_{\mathbf{x}}^{0}, \tilde{\omega}_{\mathbf{x}}\right)$ é um acoplamento clássico de $\omega_{\mathbf{x}}^{0}$ e $\omega_{\mathbf{x}}^{1}, \mathbf{x} \in \mathbb{Z}^{d}$ 
(vide [Thorisson, 2000], Capítulo 2, Seção 2, pg. 34). Sejam também $V:=\left(V_{n}\right)_{n \in \mathbb{N}}$ exponenciais padrões e $\xi:=\left(\xi_{n}\right)_{n \in \mathbb{N}}$ com distribuição $\pi$, todas independentes entre si e de todo o resto.

De acordo com a Construção II do modelo (Seção 2.2), construamos sob $\omega^{0}$ e $\tilde{\omega}$ duas versões do passeio - digamos $X^{0}:=\left(X^{0}(t)\right)_{t \in \mathbb{R}_{+}}$e $\tilde{X}:=(\tilde{X}(t))_{t \in \mathbb{R}_{+}}$, respectivamente - usando em ambas $V$ e $\xi$. Denotemos por $\mathbb{P}$ a medida de probabilidade subjacente ao par $\left(\left(\omega^{0}, X^{0}\right),(\tilde{\omega}, \tilde{X})\right)$. Ressaltamos que $X^{0}$ e $\tilde{X}$ diferem entre si apenas pelas possíveis diferenças entre $\omega^{0}$ e $\tilde{\omega}$, uma vez que possuem a mesma cadeia imersa $\mathrm{x}:=\left(\mathrm{x}_{n}\right)_{n \in \mathbb{N}}, \mathrm{x}_{n}=\sum_{i=0}^{n} \xi_{i}, n \in \mathbb{N}$; e usam das mesmas exponenciais $V$. E é claro também que $\left(\omega^{0}, X^{0}\right)$ e $(\tilde{\omega}, \tilde{X})$ constituem duas versões do processo em estudo, a primeira sob $\mathbf{P}_{\mathbf{0}}$ e a segunda sob $\mathbf{P}_{\hat{\nu}}$. Denotemos por $\left(\tau_{n}^{0}\right)_{n \in \mathbb{N}}$ e $\left(\tilde{\tau}_{n}\right)_{n \in \mathbb{N}}$ seus respectivos tempos de salto. Da construção, $\tau_{n}^{0} \leqslant \tilde{\tau}_{n}, n \in \mathbb{N}$ (detalhes na parte $b .1$ da prova da Proposição 3.1).

Agora, para $i, j \in \mathbb{N}, i \leqslant j$, façamos $\times[i, j]:=\bigcup_{k=i}^{j}\left\{\mathrm{x}_{k}\right\}, \times[i, \infty):=\lim _{l \rightarrow \infty} \times[i, l]$ e definamos

$$
\mathrm{K}_{1}=\inf \{n \in \mathbb{N}: \times[0, n] \cap \times[n+1,+\infty)=\varnothing\} .
$$

E, de modo recursivo, para $n \geqslant 2$,

$$
\mathrm{K}_{n}:=\inf \left\{n \geqslant \mathrm{~K}_{n-1}: \times[0, n] \cap \times[n+1,+\infty)=\varnothing\right\} .
$$

A sequência sequência $\left(\mathrm{K}_{n}\right)_{n \in \mathbb{N}_{*}}$ é dita tempos de corte ("cut-times", em inglês) para x e existe quase certamente uma vez que $d \geqslant 3$ e $\xi_{1}$ é limitada (vide James e Peres [1997]). Dado $L \in \mathbb{N}$, consideremos ainda $\left(\mathrm{J}_{n}\right)_{n \in \mathbb{N}}$, uma subsequência de $\left(\mathrm{K}_{n}\right)_{n \in \mathbb{N}_{*}}$, definida da seguinte maneira. Tomamos $\mathrm{J}_{1}:=\mathrm{K}_{1}$. E, de modo recursivo, para $n \geqslant 2$, façamos $\mathrm{L}_{n-1}:=\inf \left\{j \in \mathbb{N}:\left|\times\left[\mathrm{J}_{n-1}, \mathrm{~J}_{n-1}+j\right]\right|=L\right\}$ e definamos

$$
\mathrm{J}_{n}=\inf _{k \in \mathbb{N}}\left\{\mathrm{K}_{k}: \mathrm{K}_{k}>\mathrm{J}_{n-1}+\mathrm{L}_{n-1}\right\} .
$$

A partir dos elementos acima, definamos ainda

$$
\mathbf{N}=\inf _{n \in \mathbb{N}}\left\{J_{n}: \omega_{\times\left[J_{n}, J_{n}+\mathrm{L}_{n}\right]}^{0}\left(\tau_{\mathrm{J}_{n}}^{0}\right)=\tilde{\omega}_{\times\left[J_{n}, \mathrm{~J}_{n}+\mathrm{L}_{n}\right]}\left(\tilde{\tau}_{J_{n}}\right)=\mathbf{0}\right\}
$$

Das propriedades do acoplamento, temos que

$$
\begin{aligned}
& \mathbb{P}\left(\omega_{\times\left[J_{n}, J_{n}+L_{n}\right]}^{0}\left(\tau_{J_{n}}^{0}\right)=\tilde{\omega}_{\left.\times\left[J_{n},\right\lrcorner_{n}+L_{n}\right]}\left(\tilde{\tau}_{J_{n}}\right)=\mathbf{0}\right) \geqslant \mathbb{P}\left(\tilde{\omega}_{\times\left[J_{n}, J_{n}+L_{n}\right]}\left(\tau_{J_{n}}^{0}\right)=\tilde{\omega}_{\times\left[J_{n}, J_{n}+L_{n}\right]}\left(\tilde{\tau}_{J_{n}}\right)=\mathbf{0}\right) \\
& =\gamma^{L} \mathbb{P}\left(\tilde{\omega}_{\times\left[J_{n}, J_{n}+L_{n}\right]}\left(\tilde{\tau}_{J_{n}}\right)=\mathbf{0} \mid \tilde{\omega}_{\times\left[J_{n}, J_{n}+L_{n}\right]}\left(\tau_{J_{n}}^{0}\right)=\mathbf{0}\right) \\
& \geqslant \gamma^{2 L}, \quad \gamma:=1-p / q .
\end{aligned}
$$

$\mathrm{Na}$ última desigualdade usamos o fato de que as componentes do meio $\tilde{\omega}_{\left.x\left[J_{n},\right\lrcorner_{n}+\mathrm{L}_{n}\right]}$ evoluem como $\operatorname{PNM}(p, q)$ independentes em $\left[\tau_{J_{n}}^{0}, \tilde{\tau}_{\jmath_{n}}\right]$; cuja respectiva distribuição em qualquer momento nesse intervalo, a partir de uma medida inicial degenerada em $\mathbf{0}$, é (via acoplamento clássico de processos de nascimento e morte) dominada estocásticamente por $\underset{\mathbf{x} \in \times\left[J_{n}, J_{n}+\mathbf{L}_{n}\right]}{\otimes} \nu$. De (4.49), segue que

$$
\mathbb{P}\left(\mathrm{N} \geqslant \mathrm{J}_{n}\right) \leqslant\left(1-\gamma^{2 L}\right)^{n}, n \in \mathbb{N},
$$


donde decorre a finitude quase certa de N. Agora, seja $X=(X(t))_{t \in \mathbb{R}_{+}}$uma terceira versão do passeio em que

$$
X(t)=\tilde{X}(t) \mathbb{1}\left\{\tilde{\tau}_{\mathrm{N}}>t\right\}+X^{0}\left(\tau_{\mathrm{N}}^{0}+t-\tilde{\tau}_{\mathrm{N}}\right) \mathbb{1}\left\{\tilde{\tau}_{\mathrm{N}}<t\right\}
$$

Subjacente a $X$, temos o meio $\omega$, dado por

$$
\omega(t)=\tilde{\omega}(t) \mathbb{1}\left\{\tilde{\tau}_{\mathrm{N}}>t\right\}+\omega^{0}\left(\tau_{\mathrm{N}}^{0}+t-\tilde{\tau}_{\mathrm{N}}\right) \mathbb{1}\left\{\tilde{\tau}_{\mathrm{N}}<t\right\}
$$

Denotemos por $\left(\tau_{n}\right)_{n \in \mathbb{N}}$ os tempos de salto de $X$. Pela construção, para todo $t>\tau_{\mathrm{N}}=\tilde{\tau}_{\mathrm{N}}$,

$$
X(t)=X^{0}(t-U)
$$

com $U=\tilde{\tau}_{\mathrm{N}}-\tau_{\mathrm{N}}^{0}$, que é finita quase-certamente. Assim, das leis já estabelecidas para $X^{0}$, segue que, quando $t \nearrow+\infty$, para todo $\epsilon>0$,

$$
\mathbb{P}\left(\left\|\frac{X(t)}{t}\right\|>\epsilon\right) \rightarrow 0 \quad \text { e } \quad \frac{X(t)}{\sqrt{t / \mu}} \Rightarrow N_{d}(\mathbf{0}, \Sigma)
$$

com $\Sigma$ a matriz de covariância de $\xi_{1}$ e $\mu \in \mathbb{R}_{+}$.

Para findar, vamos mostrar que, em um evento de probabilidade $1-\delta_{L}$, com $\delta_{L} \rightarrow 0$ quando $L \nearrow+\infty,(\omega, X)$ coincide com uma versão do processo em estudo sob $\mathbf{P}_{\hat{\nu}}$.

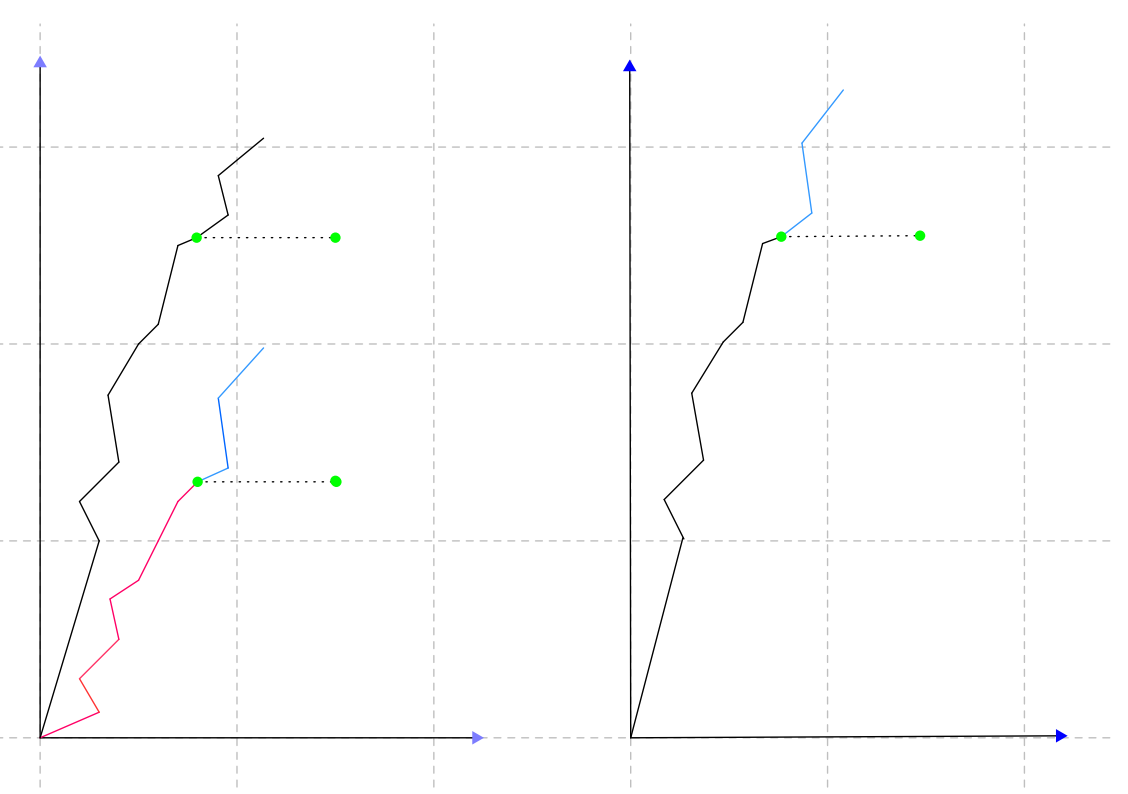

Figura 4.1: Representações de possíveis trajetórias para $X^{0}$ (em cores) e $\tilde{X}$ - ambos à esquerda - e para $X$ - à direita; com a abscissa e a ordenada representando o espaço e o tempo, respectivamente. O ponto em destaque nas trajetórias (na cor verde) representa o primeiro cut-time em que se observa o ambiente dados por zeros nos próximos destinos da partícula.

Para tal, seja $\mathrm{L}_{i}(n):=\inf \{m \geqslant n:|\times[n, m]|=i L\}, i, n \in \mathbb{N}$; definamos

$$
\kappa_{0}=\inf \left\{s>\tau_{\mathrm{N}}: X(s) \notin \times\left[\mathrm{N}, \mathrm{L}_{1}(\mathrm{~N})\right]\right\}
$$


e façamos

$$
A_{1}=\left\{\tilde{\omega}_{\times\left[\mathrm{L}_{1}(\mathrm{~N}), \mathrm{L}_{3}(\mathrm{~N})\right]}\left(\kappa_{1}-U\right)=\omega_{\times\left[\mathrm{L}_{1}(\mathrm{~N}), \mathrm{L}_{3}(\mathrm{~N})\right]}^{0}\left(\kappa_{1}-U\right)\right\} .
$$

E, de modo recursivo, para $n \geqslant 1$,

$$
\kappa_{n}=\inf \left\{s>\kappa_{n-1}: X(s) \notin \times\left[\mathrm{L}_{2^{n}-1}(\mathrm{~N}), \mathrm{L}_{2^{n+1}-1}(\mathrm{~N})\right]\right\}
$$

$\mathrm{e}$

$$
A_{n+1}=\left\{\tilde{\omega}_{\times\left[\mathrm{L}_{2^{n+1}-1}, \mathrm{~L}_{2^{n+2}-1}\right]}\left(\kappa_{n}-U\right)=\omega_{\times\left[\mathrm{L}_{2^{n+1}-1}, \mathrm{~L}_{2^{n+2}-1}\right]}^{0}\left(\kappa_{n}-U\right)\right\} .
$$

De modo análogo ao exposto em (4.37), temos que

$$
\begin{aligned}
\mathbb{P}\left(A_{1}^{c}\right) & =\int_{0}^{\infty}\left[1-\mathbb{P}\left(A_{1} \mid \kappa_{0}=s\right)\right] \mathbb{P}\left(\kappa_{0} \in \mathrm{d} s\right) \mathrm{d} s \\
& \leqslant \int_{0}^{\infty}\left[1-\left(1-\gamma e^{-\beta s}\right)^{L}\right] \mathbb{P}\left(\kappa_{0} \in \mathrm{d} s\right) \mathrm{d} s=\mathbb{E}\left(1-\left(1-\gamma e^{-\beta \kappa_{0}}\right)^{L}\right)
\end{aligned}
$$

em que $\gamma:=\sqrt{p / q}(1+\sqrt{p / q})$ e $\beta=1-2 \sqrt{p q}$. Daí, por meio de argumentos similares aos dados nas equações (4.38) e (4.39), segue que

$$
\mathbb{P}\left(A_{1}^{c}\right) \leqslant \mathbb{E}\left(\frac{2 \gamma L}{e^{\beta \kappa_{0}}}\right)+\mathbb{P}\left(e^{-\beta \kappa_{0}}>\frac{1}{2 \gamma}\right) \leqslant \mathbb{E}\left(\frac{2 \gamma L}{e^{\beta} \sum_{k=1}^{L} \mathrm{~V}_{1, i}}\right)+\mathbb{P}\left(e^{-\beta \sum_{k=1}^{L} \mathrm{~V}_{1, i}}>\frac{1}{2 \gamma}\right) \leqslant \frac{4 \gamma L}{(1+\beta)^{L}},
$$

$\operatorname{com}\left(V_{1, k}\right)_{k \in \mathbb{N}_{*}}$ uma sequência de exponenciais padrões independentes. De modo análogo, para $i \geqslant 2$, dessa vez condicionando em $\kappa_{i-1}$ e do fato de que $\kappa_{i-1} \geqslant \sum_{k=1}^{L 2^{i-1}} \mathrm{~V}_{i, k}$, com $\left\{\mathrm{V}_{i, k}\right\}_{k \in \mathbb{N}_{*}}$ exponenciais padrões independentes entre si e de todo o resto, segue

$$
\mathbb{P}\left(A_{i}^{c}\right) \leqslant \mathbb{E}\left(\frac{2^{i} \gamma L}{e^{\beta \kappa_{i-1}}}\right)+\mathbb{P}\left(e^{-\beta \kappa_{i-1}}>\frac{1}{2 \gamma}\right) \leqslant \frac{2^{(i+1)} L \gamma}{(1+\beta)^{i L}}
$$

Decorrente disso, segue que

$$
\mathbb{P}\left(\bigcap_{n \in \mathbb{N}_{*}} A_{n}\right) \geqslant 1-\sum_{i \geqslant 1} \mathbb{P}\left(A_{i}^{c}\right) \geqslant 1-\frac{C L}{(1+\beta)^{L}}
$$

para $L$ suficientemente grande, onde $C \in \mathbb{R}$ é uma constante. Mas em $\bigcap_{n \in \mathbb{N}_{*}} A_{n}$, o meio visitado por $X$ é dado por $\tilde{\omega}$, uma vez que, pós $\tau_{\mathrm{N}}$, o tempo para sair das regiões em que $\tilde{\omega}$ e $\omega$ já estão acoplados é suficiente para o acoplamento dos mesmos nas regiões subsequentes.

\subsection{Meios não ergódicos}

Todos os resultados vistos valem sob $\omega$ definido a partir de uma uma família de processos de nascimento e morte ergódicos em $S=\mathbb{N}$ independentes com taxa de nascimento $p \in(0,1 / 2)$ e taxa 
de morte $q=1-p>p$. Vamos agora supor que as componentes do meio $\left(\omega_{\mathbf{x}}(\cdot)\right)_{\mathbf{x} \in \mathbb{Z}^{d}}$ definam uma família de processos de nascimento e morte em $S=\mathbb{N}$ independentes com taxa de nascimento $p \in(1 / 2,1)$ e taxa de morte $q=1-p>p$. Temos o seguinte resultado.

Proposição 4.3. Suponhamos $p>1 / 2$. Então $X_{t} \rightarrow \mathbf{x}$ para algum $\mathbf{x} \in \mathbb{Z}^{d}$ quando $t \rightarrow \infty$, $\mathbf{P}-$ q.c.. Demonstração. Basta mostrar que $\mathbf{N}:=\sup \left\{n \in \mathbb{N}: \tau_{n}<+\infty\right\}$ é uma v.a. finita $\mathbf{P}_{\hat{\mu}_{0}}-$ q.c., $\hat{\mu}_{0}=$ $\bigotimes_{\mathbf{x} \in \mathbb{Z} d} \mu_{\mathbf{x}, 0}$ genérica. Para $n \in \mathbb{N}$, $\mathbf{x} \in \mathbb{Z}^{d}$

$$
\mathbf{P}_{\hat{\mu}_{0}}(\mathbf{N} \geqslant n)=\mathbf{P}_{\hat{\mu}_{0}}\left(\tau_{n}<+\infty\right)=\prod_{i=1}^{n} \mathbf{P}_{\hat{\mu}_{0}}\left(\Delta_{i}<+\infty \mid \tau_{i-1}<+\infty\right),
$$

com a convenção de que o produto vazio vale um. Pela propriedade forte de Markov aplicada a $\tau_{i-1}$,

$$
\mathbf{P}_{\hat{\mu}_{0}}\left(\Delta_{i}<+\infty \mid \tau_{i-1}<+\infty\right)=\mathbf{P}_{\tilde{\mu}_{0}}\left(\tau_{1}<+\infty\right),
$$

em que, sob $\tau_{i-1}<+\infty, \tilde{\mu}_{0}:=\bigotimes_{\mathbf{x} \in \mathbb{Z}^{d}} \mu_{\mathbf{x}, 0}^{i} \operatorname{com} \mu_{\mathbf{x}, 0}^{i}:=\mu_{\mathbf{x}_{i}+\mathbf{x}, \tau_{i-1}}, \mathbf{x} \in \mathbb{Z}^{d}, i \in \mathbb{N}_{*}$. Como

$$
\mathbf{P}_{\tilde{\mu}_{0}}\left(\tau_{1}<+\infty\right) \leqslant \mathbf{P}_{\mathbf{0}}\left(\tau_{1}<+\infty\right)=1-\mathbf{P}_{\mathbf{0}}\left(\tau_{1}=+\infty\right),
$$

e, pela continuidade da probabilidade,

$$
\mathbf{P}_{\mathbf{0}}\left(\tau_{1}=+\infty\right)=\lim _{k \rightarrow \infty} \mathbf{P}_{\mathbf{0}}\left(\tau_{1}>k\right)=\lim _{k \rightarrow \infty} \mathrm{E}_{\mathbf{0}}\left[\exp \left(-\int_{0}^{k} a^{-\omega_{\mathbf{0}}(s)} d s\right)\right],
$$

segue, pelo Teorema da Convergência Dominada e pela Desiguladade de Jensen, respectivamente, que

$$
\mathbf{P}_{\tilde{\mu}_{0}}\left(\tau_{1}<+\infty\right) \leqslant 1-\mathrm{E}_{\mathbf{0}}\left[\exp \left(-\int_{0}^{\infty} a^{-\omega_{\mathbf{0}}(s)} d s\right)\right] \leqslant 1-\exp \left[-\mathrm{E}_{\mathbf{0}}\left(\int_{0}^{\infty} a^{-\omega_{\mathbf{0}}(s)} \cdot d s\right)\right] .
$$

Denotemos por $\left(\mathrm{w}_{n}\right)_{n \in \mathbb{N}}$ a cadeia imersa de $\left(\omega_{\mathbf{0}}(t)\right)_{t \in \mathbb{R}_{+}}$e, para $i \in \mathbb{N}$, sejam

$$
\mathrm{H}_{i}=\inf \left\{n \in \mathbb{N}: \mathrm{w}_{n}=i\right\} \quad \text { e } \quad \mathrm{K}_{i}=\sum_{n \in \mathbb{N}} \mathbb{1}\left\{\mathrm{w}_{n}=i\right\}
$$

Façamos $\alpha_{i, j}:=\mathrm{P}_{\mathbf{i}}\left(\mathrm{H}_{j}=+\infty\right), \mathbf{i}:=i \mathbf{1}, i, j \in \mathbb{N}$. É bem sabido que $\alpha_{0}:=\alpha_{0,0}=\alpha_{1,0}=1-q / p$ e que

$$
\alpha_{i}:=\alpha_{i, i}=q \alpha_{i-1, i}+p \alpha_{i+1, i}=p \alpha_{1,0}=p \alpha_{0}, \quad i \in \mathbb{N}_{*} .
$$

Também é bem conhecido que

$$
\mathrm{P}_{\mathbf{i}}\left(\mathrm{K}_{i}=k\right)=\left(1-\alpha_{i}\right)^{k-1} \alpha_{i}, \quad k \in \mathbb{N}_{*},
$$

e é claro que

$$
\mathrm{E}_{\mathbf{0}}\left(\mathrm{K}_{i}\right) \leqslant \mathrm{E}_{\mathbf{i}}\left(\mathrm{K}_{i}\right)=\frac{1}{\alpha_{i}} \leqslant \frac{1}{p \alpha_{0}}=\frac{1}{2 p-1}, \quad i \in \mathbb{N}
$$


Temos que

$$
\mathrm{E}_{\mathbf{0}}\left(\int_{0}^{\infty} a^{-\omega_{\mathbf{0}}(s)} d s\right)=\mathrm{E}_{\mathbf{0}}\left(\sum_{i \in \mathbb{N}} a^{-i} \sum_{j=1}^{\mathrm{K}_{i}} \mathrm{~V}_{i, j}\right) \leqslant \frac{1}{p} \sum_{i \in \mathbb{N}} a^{-i} \mathrm{E}_{\mathbf{0}}\left(\mathrm{K}_{i}\right) \leqslant \frac{1}{2 p^{2}-p} \sum_{i \in \mathbb{N}} a^{-i}=: \beta<+\infty,
$$

em que $\left\{\mathrm{V}_{i, j}: i \in \mathbb{N}, j \in \mathbb{N}_{*}\right\}$ são exponenciais independentes entre si e de todo o resto, com $\left\{\bigvee_{0, j}: j \in \mathbb{N}_{*}\right\}$ com parâmetro $p$ e $\left\{\bigvee_{i, j}: i \in \mathbb{N}_{*}, j \in \mathbb{N}_{*}\right\}$ exponenciais padrões. Assim,

$$
\mathbf{P}_{\tilde{\mu}_{0}}\left(\tau_{1}<+\infty\right) \leqslant 1-e^{-\beta}, \quad \beta \in \mathbb{R}_{+},
$$

donde concluímos, pela continuidade da probabilidade, que

$$
\mathbf{P}_{\hat{\mu}_{0}}(\mathbf{N}=+\infty)=\lim _{n \nearrow+\infty} \mathbf{P}_{\hat{\mu}_{0}}(\mathbf{N} \geqslant n) \leqslant \lim _{n \nearrow+\infty}\left(1-e^{-\beta}\right)^{n}=0, \quad \beta \in \mathbb{R}_{+} .
$$

É fácil ver que tal resultado permanece válido $\operatorname{com} \omega_{\mathbf{x}}, \mathbf{x} \in \mathbb{Z}^{d}$, não identicamente distribuídos, isto é, independentes, transientes, mas não necessariamente com as mesmas taxas. Além disso, ressaltamos que para o caso simétrico com $d \leqslant 2$, a localização ocorre ainda que apenas um dos processos de nascimento e morte seja transiente e os demais ergódicos. Isso segue da recorrência do passeio para $d \leqslant 2$ e da probabilidade positiva de localização em cada passagem pela componente transitória.

No caso $p=1 / 2, q=1 / 2$, assim como no caso $p<1 / 2, \tau_{n}<+\infty, \mathbf{P}_{\hat{\mu}_{0}}-q . c ., n \in \mathbb{N}$. De fato, por indução, $\tau_{0} \equiv 0<+\infty$ e, supondo $\tau_{n}<+\infty, n \in \mathbb{N}$,

$$
\mathbf{P}_{\hat{\mu}_{0}}\left(\Delta_{n+1}=+\infty\right) \leqslant \mathrm{E}_{\hat{\mu}_{0}}\left[\exp \left(-\int_{\tau_{n}}^{\infty} \mathbb{1}\left\{\omega_{x_{n}}(s)=0\right\} d s\right)\right]=0,
$$

uma vez que a integral no termo central da expressão acima é infinita $\mathrm{P}_{\hat{\mu}_{0}}$ - q.c., pela recorrência de $\omega_{x_{n}}$. Assim, diferente do caso $p>1 / 2$, a partícula continua a performar saltos ao longo do tempo $\mathbf{P}_{\hat{\mu}_{0}}-$ q.c.. Por outro lado, esperamos que $\mathbf{E}\left(\Delta_{n}\right)=\infty$ para todo $n \in \mathbb{N}_{*}$ e que $X$ seja sub-difusivo. Nesse sentido, $p=1 / 2$ seria um ponto de transição de fase em relação ao comportamento assintótico da partícula. 


\section{Capítulo 5}

\section{Conclusões}

Vimos que para $p<1 / 2$, qualquer que seja $a>1$, vale um Teorema Central do Limite para a posição da partícula partir da medida inicial degenerada em 0 para o meio $\omega$. Posteriormente, mostramos que os resultados permanecem, com probabilidade tão alta quanto se queira, para meios com medida inicial dominada estocasticamente pela medida produto de distribuições geométricas independentes, todas com parâmetro $\rho=p / q$. Por fim, inciamos a discussão sobre o caso em que $\omega$ é dado por componentes não ergódicas: mostramos a localização do passeio para $\omega$ transiente e conjeturamos um comportamento sub-difusivo para a partícula sob $\omega$ recorrente nulo.

Além de Teoremas Limites sob $\omega$ recorrente nulo, muitas outras perguntas sobre o modelo permanecem. A primeira consiste de uma extensão dos resultados para um passeio de média nãonula. Uma possível solução para esta talvez possa ser consequência de uma medida limite para o meio visto pela partícula, cuja existência consiste numa segunda questão. Também pode-se pensar sobre a inserção de alguma dependência nos meios e/ou entre as direções da partícula e o meio em si; e a possibilidade várias partículas no meio sob alguma regra de interação (exclusão simples, por exemplo). 


\section{Referências Bibliográficas}

Avena(2010) Luca Avena. Random walks in dynamic random environments. Tese de Doutorado, Leiden University, Netherlands. URL https://hdl.handle.net/1887/16072. Citado na pág. 1

Bandyopadhyay e Zeitouni(2006) Antar Bandyopadhyay e Ofer Zeitouni. Random walks in dynamic markovian random environment. Alea, 1:205-224. Citado na pág. 1

Boldrighini et al.(1992) C. Boldrighini, I. A. Ignatyuk, V. A. Malyshev e A. Pellegrinotti. Random walk in dynamic environment with mutual influence. Stochastic Processes and their Applications, 41(1):157-177. ISSN 0304-4149. doi: http://dx.doi.org/10.1016/0304-4149(92)90151-F. URL http://www.sciencedirect.com/science/article/pii/030441499290151F. Citado na pág. 1

Boldrighini et al.(2007) Carlo Boldrighini, Robert A Minlos e Alessandro Pellegrinotti. Random walks in a random (fluctuating) environment. Russian Mathematical Surveys, 62(4):663-712. URL http://stacks.iop.org/0036-0279/62/i=4/a=R02. Citado na pág. 1

Dolgopyat e Liverani(2009) Dmitry Dolgopyat e Carlangelo Liverani. Non-perturbative approach to random walk in markovian environment. Electron. Commun. Probab., 14:245-251. doi: 10.1214/ECP.v14-1467. URL http://dx.doi.org/10.1214/ECP.v14-1467. Citado na pág. 1, 2

Dolgopyat et al.(2008) Dmitry Dolgopyat, Gerhard Keller e Carlangelo Liverani. Random walk in markovian environment. Ann. Probab., 36(5):1676-1710. doi: 10.1214/07-AOP369. URL http://dx.doi.org/10.1214/07-AOP369. Citado na pág. 1, 2

Durret(2010) Richard Durret. Probability: theory and examples. Cambridge University Press, quarta edição. Citado na pág. 2, 7, 17, 18, 23

Durrett et al.(1991) R. Durrett, H. Kesten e G. Lawler. Making Money From Fair Games, páginas 255-267. Birkhäuser Boston, Boston, MA. ISBN 978-1-4612-0459-6. doi: 10.1007/ 978-1-4612-0459-6_13. URL https://doi.org/10.1007/978-1-4612-0459-6_13. Citado na pág. 31

Fontes et al.(2002) L. R. G. Fontes, M. Isopi e C. M. Newman. Random walks with strongly inhomogeneous rates and singular diffusions: convergence, localization and aging in one dimension. Ann. Probab., 30(2):579-604. doi: 10.1214/aop/1023481003. URL http://dx.doi.org/10.1214/ aop/1023481003. Citado na pág. 5

James e Peres(1997) N. James e Y. Peres. Cutpoints and exchangeable events for random walks. Theory of Probability \& Its Applications, 41(4):666-677. doi: 10.1137/S0040585X97975745. URL https://doi.org/10.1137/S0040585X97975745. Citado na pág. 32

Lima(2010) Elon Lages Lima. Curso de análise, volume 1. IMPA, décima segunda edição. Citado na pág. 6,12

Norris(1997) J. R. Norris. Markov Chains. Cambridge University Press. Citado na pág. 10

Redig e Völlering(2013) Frank Redig e Florian Völlering. Random walks in dynamic random environments: a transference principle. Ann. Probab., 41(5):3157-3180. doi: 10.1214/12-AOP819. URL http://dx.doi.org/10.1214/12-AOP819. Citado na pág. 1, 2 
Spitzer(1976) Frank Spitzer. Principles of Random Walk. Graduate texts in mathematics. Springer-Verlag New York. Citado na pág. 1, 28

Thorisson(2000) Hermann Thorisson. Coupling, Stationarity, and Regeneration. Probability and Its Applications. Springer New York. Citado na pág. 10, 17, 32

Yilmaz(2009) Atilla Yilmaz. Large deviations for random walk in a space-time product environment. Ann. Probab., 37(1):189-205. doi: 10.1214/08-AOP400. URL http://dx.doi.org/10.1214/ 08-AOP400. Citado na pág. 1 